\title{
SUPERSYMMETRY, TWISTORS, AND THE YANG-MILLS EQUATIONS
}

\author{
MICHAEL EASTWOOD
}

\begin{abstract}
This article investigates a supersymmetric proof due to Witten of the twistor description of general Yang-Mills fields due to Green, Isenberg, and Yasskin. In particular, some rigor is added and the rather complicated calculations are given in detail.
\end{abstract}

0. Introduction. In a remarkable paper Witten [24] indicated that there is a close connection between the classical Yang-Mills equations and a certain system of supersymmetric equations arising from Penrose's twistor theory $[\mathbf{1 6}, \mathbf{1 7}]$ as modified by Ferber $[\mathbf{7}]$. In particular, he uses this connection to give a rather natural proof of the theorem of Green, Isenberg, and Yasskin [9] concerning the twistor description of the full Yang-Mills equations. For alternative approaches to this theorem see Manin [14], Pool [19], or Buchdahl [2].

This article has two objectives. The first is to provide, in reasonable detail, the rather involved calculations omitted from [24]. These calculations are highly complicated in spite of the apparently simple conclusions. The seemingly miraculous cancelations which occur are reminiscent of similar cancelations found in the renormalization of supersymmetric field theories.

The second objective is to impart a little more rigor to the arguments. Although written for mathematicians, this article proceeds in an informal manner during $\S \S 1-5$ where the notation is established and the main theorem is stated (in $\S 5$ ). This is entirely reasonable and standard practice in physics, and the hardened mathematician is requested to suspend disbelief until $\S 6$ where precision is restored. In order not to interrupt the flow of argument, the detailed calculations alluded to earlier are reserved for an appendix ( $§ 7)$.

1. Superspace. Roughly speaking, a supermanifold consists of an underlying smooth manifold $Q$ together with some anticommuting variables $y^{\alpha}$ for $\alpha=1, \ldots, n$. In general there is no special relation between $n$ and the dimension of $Q$. More precisely, these local anticommuting variables are allowed to change over $Q$ in order to define a vector bundle. For the moment all such subtleties are suspended until $\S 6$. Locally, a superfunction (i.e. a "function" on a supermanifold) can be expanded as a power series in the anticommuting variables:

$$
F(x, y)=f(x)+y^{\alpha} f_{\alpha}(x)+\cdots+y^{\alpha} y^{\beta} \cdots y^{\gamma} f_{\alpha \beta \cdots \gamma}(x)+\cdots .
$$

Here, the Einstein summation convention has been used and the resulting coefficients are skew in their indices--i.e., $f_{\alpha \beta \cdots \gamma}=f_{[\alpha \beta \cdots \gamma]}$ (brackets are used to denote

Received by the editors October 16, 1984.

1980 Mathematics Subject Classification (1985 Revision). Primary 53C80; Secondary 53A50, 32 C35.

Research supported by an S.E.R.C. Advanced Fellowship. 
antisymmetrization, and parentheses symmetrization), and it follows that this expansion is finite, automatically truncating at order $n$. There is a parallel construction in the holomorphic category.

Superspace is an example of a holomorphic supermanifold where the underlying manifold is complexified Minkowski space $M$ with coordinates $x^{a}=x^{A A^{\prime}}$ (see $[\mathbf{1 6}$, 18] for this notation). $M_{[N]}$, superspace of $\operatorname{order} N$, is that supermanifold formed by adjoining $4 N$ anticommuting variables $\theta_{j}^{A}$ and $\tilde{\theta}^{A^{\prime} j}$ for $j=1, \ldots, N$. The indices $A$ and $A^{\prime}$ are genuine spinor indices (see [18] for a full discussion of spinors using this notation).

One can do calculus on supermanifolds provided care is taken with ordering derivatives. Standard results and constructions from classical calculus on manifolds such as the Leibnitz rule, the Frobenius integrability theorem, and the de Rham sequence generalize to the category of supermanifolds. Of particular interest are the following differential operators on $M_{[N]}$ :

$$
\partial_{A}^{j}=\frac{\partial}{\partial \theta_{j}^{A}}+\tilde{\theta}^{A^{\prime} j} D_{A A^{\prime}} \quad \text { and } \quad \tilde{\partial}_{A^{\prime} j}=\frac{\partial}{\partial \tilde{\theta}^{A^{\prime} j}}+\theta_{j}^{A} D_{A A^{\prime}}
$$

where $D_{a}=\partial / \partial x^{a}$. They satisfy the following (anti)commutation relations:

$$
\left[\partial_{A}^{j}, D_{b}\right]=0=\left[\tilde{\partial}_{A^{\prime} j}, D_{b}\right], \quad\left(\partial_{A}^{j}, \partial_{B}^{k}\right)=0=\left(\tilde{\partial}_{A^{\prime} j}, \tilde{\delta}_{B^{\prime} k}\right), \quad\left(\partial_{A}^{j}, \tilde{\partial}_{A^{\prime} k}\right)=2 \delta_{k}^{j} D_{A A^{\prime}}
$$

where [, ] denotes a commutator and $($, ) an anticommutator. Along with the usual $\left[D_{a}, D_{b}\right]=0$ these relations define a superalgebra often taken as the motivation for introducing superspace [20].

2. Superambitwistors. Twistor space $T$ is a 4-dimensional complex vector space with coordinates traditionally denoted $Z^{\alpha}=\left(\omega^{A}, \pi_{A^{\prime}}\right)$ (for further explanation of this notation see [16]). Coordinates for the dual shall be denoted $W_{\alpha}=\left(\xi^{A^{\prime}}, \eta_{A}\right)$. Ambitwistor space (see $\left.[\mathbf{3}]\right) A$ is defined to be

$$
A=\left\{(Z, W) \in \mathrm{PT} \times \mathrm{PT}^{*} \text { s.t. } Z^{\alpha} W_{\alpha}=0\right\} .
$$

Here PT means projective twistor space. The usual twistor correspondence [16, 22, 23] with Minkowski space has a counterpart for ambitwistors defined by the incidence relations

$$
\omega^{A}=x^{A A^{\prime}} \pi_{A^{\prime}}, \quad \xi^{A^{\prime}}=-x^{A A^{\prime}} \eta_{A} .
$$

For each $x \in M$ these relations define a quadric $Q_{x}$ in $A$, whereas each point of $A$ represents a null geodesic or light ray in $M$. This is the basic correspondence used by Green, Isenberg, and Yasskin [9].

Superambitwistor space of order $N$ as introduced by Witten [24] involves adjoining $2 N$ extra anticommuting variables. It has homogeneous coordinates

$$
\left[Z^{\alpha}, W_{\alpha}, \zeta^{j}, \psi_{j}\right] \text { for } j=1, \ldots, N
$$

s.t.

$$
\left[Z^{\alpha}, W_{\alpha}, \varsigma^{j}, \psi_{j}\right]=\left[\lambda Z^{\alpha}, \mu W_{\alpha}, \lambda \varsigma^{j}, \mu \psi_{j}\right] \quad \text { for nonzero } \lambda \text { and } \mu
$$

and

$$
Z^{\alpha} W_{\alpha}=2 \zeta^{j} \psi_{j}
$$


There is a correspondence with superspace $M_{[N]}$ defined by the relations

$$
\begin{aligned}
\omega^{A} & =\left(x^{A A^{\prime}}-\theta_{j}^{A} \tilde{\theta}^{A^{\prime} j}\right) \pi_{A^{\prime}}, \quad \xi^{A^{\prime}}=\left(-x^{A A^{\prime}}-\theta_{j}^{A} \tilde{\theta}^{A^{\prime} j}\right) \eta_{A}, \\
\zeta^{j} & =\tilde{\theta}^{A^{\prime} j} \pi_{A^{\prime}}, \quad \psi_{j}=\theta_{j}^{A} \eta_{A} .
\end{aligned}
$$

Each point $(x, \theta, \tilde{\theta}) \in M_{[N]}$ still represents a quadric $Q_{(x, \theta, \tilde{\theta})}$ in $A_{[N]}$, but now each point of $A_{[N]}$ defines a superlight ray in $M_{[N]}$, a supermanifold with one ordinary dimension but $2 N$ additional anticommuting variables. As Witten points out, it is these extra dimensions which give rise to interesting equations, since integrability of a connection along a superlight ray is no longer a trivial condition for $N>0$.

3. Thickambitwistors. As a submanifold of $\mathrm{PT} \times \mathrm{PT}^{*}, A$ may be thickened out to its $N$ th formal neighborhood (see $[2,8]$ ). Precisely, $A_{(N)}$ is defined to be $A$ but with an enlarged sheaf of holomorphic functions

$$
O_{(N)}=O_{\mathrm{PT} \times \mathrm{PT}^{*}} / I^{N+1}
$$

where $I$ is the ideal sheaf of $A$ inside $\mathrm{PT} \times \mathrm{PT}^{*}$. More informally, however, thickambitwistors $A_{(N)}$ may be regarded as formed by adjoining an extra commuting variable whose $(N+1)$ st power vanishes. It has homogeneous coordinates $\left[Z^{\alpha}, W_{\alpha}, \chi\right]$ s.t.

$$
\left[Z^{\alpha}, W_{\alpha}, \chi\right]=\left[\lambda Z^{\alpha}, \mu W_{\alpha}, \lambda \mu \chi\right], \quad Z^{\alpha} W_{\alpha}=\chi, \quad \text { and } \quad \chi^{N+1}=0 .
$$

The theorem of [9] may now be stated:

TheOREM (GREen, ISENBERG, AND YASSKIN). For $U$ an open subset of $M$, let $Q_{U(N)}$ denote the corresponding region of $A_{(N)}$ swept out by $Q_{x}$ for $x \in U$. Suppose every light ray in $U$ is simply connected (and, in particular, connected). Then there is a 1-1 correspondence between solutions of the holomorphic YangMills equations on $U$ and holomorphic vector bundles on $Q_{U(3)}$ trivial on each $Q_{x}$ for $x \in U$.

Since $\varsigma^{j}$ and $\psi_{j}$ are anticommuting variables, it follows that $\left(\varsigma^{j} \psi_{j}\right)^{N+1}=0$. Therefore there is a map $A_{[N]} \rightarrow A_{(N)}$ defined by $\chi=2 \varsigma^{j} \psi_{j}$. Indeed there is the commutative diagram

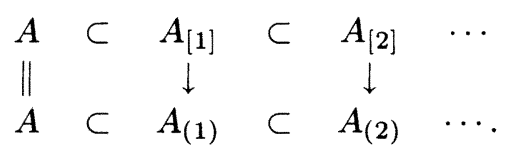

This link suggests introducing thickspace $M_{(N)}$ by adjoining $\sigma^{a}=\theta_{j}^{A} \tilde{\theta}^{A^{\prime} j}$. There is then a correspondence between $A_{(N)}$ and $M_{(N)}$ defined by the incidence relations

$$
\omega^{A}=\left(x^{A A^{\prime}}-\sigma^{A A^{\prime}}\right) \pi_{A^{\prime}}, \quad \xi^{A^{\prime}}=\left(-x^{A A^{\prime}}-\sigma^{A A^{\prime}}\right) \eta_{A}, \quad \chi=-2 \sigma^{A A^{\prime}} \eta_{A} \pi_{A^{\prime}}
$$

The anticommuting nature of the variables $\theta_{j}^{A}$ and $\tilde{\theta}^{A^{\prime} j}$ implies certain constraints on the variables $\sigma^{a}$. For example, in case $N=1$, although $\sigma^{a}$ and $\sigma^{b}$ commute, one has

$$
\sigma^{a} \sigma^{b}=\frac{1}{4} \varepsilon^{A B} \varepsilon^{A^{\prime} B^{\prime}}\left(\sigma^{c} \sigma^{d} \varepsilon_{C D} \varepsilon_{C^{\prime} D^{\prime}}\right) .
$$

In other words, although one would expect, in the spinor decomposition of $\sigma^{a} \sigma^{b}$, a term of the form $\alpha^{A B A^{\prime} B^{\prime}}=\alpha^{(A B)\left(A^{\prime} B^{\prime}\right)}$, this part has been set to zero. For 
$N=1$ all higher powers of $\sigma^{a}$ vanish. For $N>1$ there are similar but more complicated constraints (see $\S 7$ for details). Thus, although $M_{(N)}$ is an unreduced analytic space in the classical sense, it is not simply a formal neighborhood of $M$ as the diagonal in $M \times M$. As for $A$, there is a commutative diagram

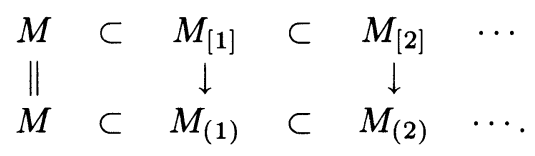

One can introduce differential operators $\delta_{a}$ and $\tilde{\delta}_{a}$ on $M_{(N)}$ by $\delta_{a}=D_{a}+E_{a}$ and $\tilde{\delta}_{a}=D_{a}-E_{a}$ where $E_{a}=\partial / \partial \sigma^{a}$ (for calculus on an analytic space see [2]). Then, if a thickfunction is regarded via $\sigma^{a}=\theta_{j}^{A} \tilde{\theta}^{A^{\prime} j}$ as a special case of a superfunction,

$$
\partial_{A}^{j}=\tilde{\theta}^{A^{\prime} j} \delta_{A A^{\prime}} \quad \text { and } \quad \tilde{\partial}_{A^{\prime} j}=\theta_{j}^{A} \delta_{A A^{\prime}}
$$

4. The Ward correspondence. Ward's interpretation $[\mathbf{2 1}]$ of self-dual gauge fields via twistors was extended by Green, Isenberg, and Yasskin [9] to arbitrary gauge fields in terms of ambitwistors and by Witten [24] to superfields using superambitwistors. A common feature of these correspondences is that the argument for the Abelian case (e.g. Ward's "nonlinear photon" construction) goes through with essentially no change for the general case. All that is involved is the interpretation of potential/gauge (see e.g. [17]) as a connection, and so on. However, in order not to become involved in defining all the relevant concepts in the category of supermanifolds, it is easier to stick to the potential/gauge description and the Abelian case. This also allows one to use the Sparling-Ward "splitting method" $[\mathbf{1 7}]$ for the correspondence (which may be regarded, for the Abelian gauge group, as a special case of the Penrose transform $[4,5])$. At only one point in $\S 5$ is there any need for provisos concerning the generalization. Such provisos will be clearly stated, but all remaining arguments in this article will be given for the Abelian case only (i.e., for connections on a trivial line bundle).

To minimize notation, an open subset of $M$ will also be denoted by $M$ and will always be assumed to satisfy mild topological restrictions-namely, that each light ray be simply connected. The corresponding subset of $A$ swept out by $Q_{x}$ for $x \in M$ will be denoted by $A$ too. Discussion of the Penrose-Ward transform will be confined to describing the direction from $A$ to $M$. Proving that this results in an isomorphism is accomplished along now standard lines $[\mathbf{4}, \mathbf{5}]$ and will be omitted.

To introduce the results for $N>0$, first recall $[\mathbf{1 7}, \mathbf{4}, \mathbf{5}]$ the Penrose-Ward transform of $H^{1}(A, 0)$. To effect this by the Sparling-Ward splitting method, regard a cohomology class by means of a Čech cocycle $F_{\alpha \beta}(Z, W)$ with respect to some suitable cover. Using the ambitwistor incidence relations form

$$
f_{\alpha \beta}(x, \pi, \eta)=F_{\alpha \beta}\left(x^{A A^{\prime}} \pi_{A^{\prime}}^{\prime}, \pi_{A^{\prime}},-x^{A A^{\prime}} \eta_{A^{\prime}} \eta_{A}\right) .
$$

Since $H^{1}\left(Q_{x}, 0\right)=0$ for each $x \in M$, the class $f_{\alpha \beta}$ is cohomologous to zero and therefore splits as $f_{\alpha \beta}=f_{\alpha}(x, \pi, \eta)-f_{\beta}(x, \pi, \eta)$. Now the form of $f_{\alpha \beta}$ implies that

$$
\eta^{A} \pi^{A^{\prime}} D_{A A^{\prime}} f_{\alpha \beta}=\eta^{A} \pi^{A^{\prime}}\left(\pi_{A^{\prime}} \frac{\partial F_{\alpha \beta}}{\partial \omega^{A}}-\eta_{A} \frac{\partial F_{\alpha \beta}}{\partial \xi^{A^{\prime}}}\right)=0
$$


so $\eta^{A} \pi^{A^{\prime}} D_{A A^{\prime}} f_{\alpha}$ is globally defined in $\pi$ and $\eta$. It is also homogeneous of degree $(1,1)$ and therefore must be a polynomial

$$
\eta^{A} \pi^{A^{\prime}} D_{A A^{\prime}} f_{\alpha}=\eta^{A} \pi^{A^{\prime}} \Phi_{A A^{\prime}}(x) .
$$

This defines the potential $\Phi_{a}$ on $M$, whereas a different choice of splitting provides the gauge freedom $\Phi_{a} \mapsto \Phi_{a}+D_{a} g$. This standard argument generalizes easily to deal with $H^{1}\left(A_{[N]}, 0\right)$. The superambitwistor correspondence gives

$$
\begin{aligned}
& f_{\alpha \beta}(x, \theta, \tilde{\theta}, \pi, \eta) \\
& \quad=F_{\alpha \beta}\left(\left(x^{A A^{\prime}}-\sigma^{A A^{\prime}}\right) \pi_{A^{\prime}}, \pi_{A^{\prime}},\left(-x^{A A^{\prime}}-\sigma^{A A^{\prime}}\right) \eta_{A}, \eta_{A}, \tilde{\theta}^{A^{\prime} j} \pi_{A^{\prime}}, \theta_{j}^{A} \eta_{A}\right) .
\end{aligned}
$$

which satisfies the differential equations

$$
\eta^{A} \partial_{A}^{j} f_{\alpha \beta}=0, \quad \pi^{A^{\prime}} \tilde{\partial}_{A^{\prime} j} f_{\alpha \beta}=0, \quad \eta^{A} \pi^{A^{\prime}} D_{A A^{\prime}} f_{\alpha \beta}=0 .
$$

Thus, after splitting, there are "potentials" $\Psi_{A}^{j}(x, \theta, \tilde{\theta}), \tilde{\Psi}_{A^{\prime} j}(x, \theta, \tilde{\theta})$, and $\Phi_{a}(x, \theta, \tilde{\theta})$ given by

$$
\eta^{A} \partial_{A}^{j} f_{\alpha}=\eta^{A} \Psi_{A}^{j}, \quad \pi^{A^{\prime}} \tilde{\partial}_{A^{\prime} j} f_{\alpha}=\pi^{A^{\prime}} \tilde{\Psi}_{A^{\prime} j}, \quad \eta^{A} \pi^{A^{\prime}} D_{A A^{\prime}} f_{\alpha}=\eta^{A} \pi^{A^{\prime}} \Phi_{A A^{\prime}}
$$

and defined up to gauge freedom $\Psi_{A}^{j} \mapsto \Psi_{A}^{j}+\partial_{A}^{j} g, \tilde{\Psi}_{A^{\prime} j} \mapsto \tilde{\Psi}_{A^{\prime} j}+\tilde{\partial}_{A^{\prime} j} g$, and $\Phi_{a} \mapsto \Phi_{a}+D_{a} g$ for any superfunction $g(x, \theta, \tilde{\theta})$. These potentials are not arbitrary, however. Since $\eta^{B} \partial_{B}^{k} \Psi_{A}^{j}=\eta^{A} \eta^{B} \partial_{B}^{k} \partial_{A}^{j} f_{\alpha}$ and $\left(\partial_{B}^{k}, \partial_{A}^{j}\right)=0$, it follows that $\partial_{(A}^{(j} \Psi_{B)}^{k)}=0$. There is a similar equation on $\tilde{\Psi}_{A^{\prime} j}$, and finally, since $\left(\partial_{A}^{j} \tilde{\partial}_{A^{\prime} k}\right)=2 \delta_{k}^{j} D_{a}$, one obtains $\partial_{A}^{j} \tilde{\Psi}_{A^{\prime} k}+\tilde{\partial}_{A^{\prime} k} \Psi_{A}^{j}=2 \delta_{k}^{j} \Phi_{a}$. With standard arguments alluded to earlier this proves the following

THEOREM (WITTEN). $H^{1}\left(A_{[N]}, 0\right)$ is isomorphic to the space of "potentials" $\Psi_{A}^{j}, \tilde{\Psi}_{A^{\prime} j}$, and $\Phi_{a}$ on $M_{[N]}$ satisfying

$\partial_{(A}^{j} \Psi_{B)}^{k}+\partial_{(A}^{k} \Psi_{B)}^{j}=0, \quad \tilde{\partial}_{j\left(A^{\prime}\right.} \tilde{\Psi}_{\left.B^{\prime}\right) k}+\tilde{\partial}_{k\left(A^{\prime}\right.} \tilde{\Psi}_{\left.B^{\prime}\right) j}=0, \quad \partial_{A}^{j} \tilde{\Psi}_{A^{\prime} k}+\tilde{\partial}_{A^{\prime} k} \Psi_{A}^{j}=2 \delta_{k}^{j} \Phi_{a}$ and defined up to gauge frredom of $\Psi_{A}^{j} \mapsto \Psi_{A}^{j}+\partial_{A}^{j} g, \tilde{\Psi}_{A^{\prime} j} \mapsto \tilde{\Psi}_{A^{\prime} j}+\tilde{\partial}_{A^{\prime} j} g$, and $\Phi_{a} \mapsto \Phi_{a}+D_{a} g$ for an arbitrary superfunction $g=g(x, \theta, \tilde{\theta})$.

If $f_{\alpha \beta}$ is actually a thickambitwistor function rather than just a superambitwistor function-i.e., it represents an element of $H^{1}\left(A_{(N)}, 0\right)$-then the resulting potentails satisfy further restrictions. To identity these restrictions recall (§3) that, for thickfunctions, $\partial_{A}^{j}=\tilde{\theta}^{A^{\prime} j} \delta_{A A^{\prime}}$, so $\tilde{\theta}^{A^{\prime} j} \eta^{A} \delta_{A A^{\prime}} f_{\alpha \beta}=\eta^{A} \partial_{A}^{j} f_{\alpha \beta}=0$. It is not the case, however, that $\eta^{A} \delta_{A A^{\prime}} f_{\alpha \beta}$ necessarily vanishes. For example, if $N=1$ and

$$
F_{\alpha \beta}(Z, W, \chi)=\omega^{A} \omega^{B} K_{A B} / L^{A^{\prime} B^{\prime}} \pi_{A^{\prime}} \pi_{B^{\prime}}
$$

for constant spinors $K_{A B}=K_{(A B)}$ and $L^{A^{\prime} B^{\prime}}=L^{\left(A^{\prime} B^{\prime}\right)}$, then

$$
\begin{aligned}
f_{\alpha \beta} & =(x-\sigma)^{a}(x-\sigma)^{b} K_{A B} \pi_{A^{\prime}} \pi_{B^{\prime}} / L^{A^{\prime} B^{\prime}} \pi_{A^{\prime}} \pi_{B^{\prime}} \\
& =\left(x^{a} x^{b}-2 \sigma^{a} x^{b}\right) K_{A B} \pi_{A^{\prime}} \pi_{B^{\prime}} / L^{A^{\prime} B^{\prime}} \pi_{A^{\prime}} \pi_{B^{\prime}},
\end{aligned}
$$

so

$$
\begin{aligned}
\eta^{A} \delta_{A A^{\prime}} f_{\alpha \beta} & =\eta^{A}\left(2 x^{b}-2 \sigma^{b}-2 x^{b}\right) K_{A B} \pi_{A^{\prime}} \pi_{B^{\prime}} / L^{A^{\prime} B^{\prime}} \pi_{A^{\prime}} \pi_{B^{\prime}} \\
& =-2 \eta^{A} \theta^{B} K_{A B} \tilde{\theta}^{B^{\prime}} \pi_{A^{\prime}} \pi_{B^{\prime}} / L^{A^{\prime} B^{\prime}} \pi_{A^{\prime}} \pi_{B^{\prime}}
\end{aligned}
$$


Nevertheless it is the case that $\tilde{\theta}^{A^{\prime}} \eta^{A} \delta_{A A^{\prime}} f_{\alpha \beta}=0$ since $\tilde{\theta}^{A^{\prime}} \tilde{\theta}^{B^{\prime}}$ is skew in $A^{\prime}, B^{\prime}$, whereas $\pi_{A^{\prime}} \pi_{B^{\prime}}$ is symmetric. This example brings to light an earlier subtlety which was skimmed over, namely that it is not completely obvious that $\eta^{A} \partial_{A}^{j} f_{\alpha \beta}=0$ for $f_{\alpha \beta}$ created from a superambitwistor function $F_{\alpha \beta}$. Nevertheless it is elementary to check that this is indeed the case by expanding $f_{\alpha \beta}$ as a power series. This difficulty also illustrates why it is necessary to introduce superambitwistors. It might at first be thought that a naive approach using the thickcorrespondence might interpret $H^{1}\left(A_{(N)}, 0\right)$ on $M_{(N)}$, but such a method would require $\eta^{A} \delta_{A A^{\prime}} f_{\alpha \beta}$ to vanish. In spite of this problem we can still conclude:

LEMMA. Starting with a cohomology class in $H^{1}\left(A_{(N)}, 0\right)$ the corresponding Witten potential $\Psi_{A}^{j}$ may be chosen to have the form $\Psi_{A}^{j}=\tilde{\theta}^{A^{\prime} j} \Sigma_{A A^{\prime}}$ for $\Sigma_{a}=$ $\Sigma_{a}(x, \sigma)$.

PROOF. Consider $\eta^{A} \delta_{A A^{\prime}} f_{\alpha \beta}$ as a power series expanded in the variables $\sigma$ :

$$
\eta^{A} \delta_{A A^{\prime}} f_{\alpha \beta}=p_{A^{\prime}}(x, \pi, \eta)+\sigma^{b} p_{A^{\prime} b}(x, \pi, \eta)+\sigma^{b} \sigma^{c} p_{A^{\prime} b c}(x, \pi, \eta)+\cdots
$$

As remarked earlier, these variables enjoy certain symmetries forced by the anticommuting nature of $\theta_{j}^{A}$ and $\tilde{\theta}^{A^{\prime} \jmath}$. For example, this series necessarily terminates. More precisely, if each coefficient is decomposed into irreducible spinor parts (see [18]), then many of these parts do not contribute (because they conflict with the symmetries of the variables $\sigma^{a}$ ). If $N=1$ for example, then one may take

$$
\begin{aligned}
& p_{A^{\prime}}=p_{A^{\prime}}, \\
& p_{A^{\prime} b}=\varepsilon_{A^{\prime} B^{\prime}} q_{B}+r_{A^{\prime} B^{\prime} B} \quad \text { where } r_{A^{\prime} B^{\prime} B}=r_{\left(A^{\prime} B^{\prime}\right) B}, \\
& p_{A^{\prime} b c}=s_{A^{\prime}} \varepsilon_{B C} \varepsilon_{B^{\prime} C^{\prime}},
\end{aligned}
$$

and all other coefficients zero. The case $N=2$ is worked out in detail in $\S 7$, but for this proof the details are unimportant. Contracting with $\tilde{\theta}^{A^{\prime} j}$ now has the effect (by Schur's lemma) of preserving some of these irreducible parts while eliminating others. Again to take the case $N=1$ :

$$
\tilde{\theta}^{A^{\prime}} \eta^{A} \delta_{A A^{\prime}} f_{\alpha \beta}=\tilde{\theta}^{A^{\prime}} p_{A^{\prime}}(x, \pi, \eta)+\tilde{\theta}^{A^{\prime}} \sigma^{b} \varepsilon_{A^{\prime} B^{\prime} B}(x, \pi, \eta) .
$$

Hence, the vanishing of $\tilde{\theta}^{A^{\prime}} \eta^{A} \delta_{A A^{\prime}} f_{\alpha \beta}$ means precisely that these surviving coefficients must vanish. Thus one can apply the Sparling-Ward splitting method to these coefficients in order to define the corresponding parts of a $\sigma$ power series expansion of $\Sigma_{a}$. Note that

$$
\eta^{A} \tilde{\theta}^{A^{\prime} j} \Sigma_{A A^{\prime}}=\eta^{A} \partial_{A}^{j} f=\eta^{A} \Psi_{A}^{j}
$$

because $\Sigma$ only appears in conjunction with $\tilde{\theta}^{A^{\prime} j}$, and it is exactly the coefficients for $\tilde{\theta}^{A^{\prime} j} \Sigma_{A A^{\prime}}$ which have been determined by the splitting method. Thus $\Psi_{A}^{j}=$ $\tilde{\theta}^{A^{\prime} j} \Sigma_{A A^{\prime}}$ as required.

Similarly, $\tilde{\Psi}_{A^{\prime} j}$ may be taken to have the form $\theta_{j}^{A} \tilde{\Sigma}_{A A^{\prime}}(x, \sigma)$. The equations of Witten's theorem may be phrased in terms of $\Sigma_{a}$ and $\tilde{\Sigma}_{a}$ with restricted gauge freedom $\Sigma_{a} \mapsto \Sigma_{a}+\delta_{a} g$ and $\tilde{\Sigma}_{a} \mapsto \tilde{\Sigma}_{a}+\tilde{\delta}_{a} g$ for any thickfunction $g(x, \sigma)$. However, one can specialize a little further by insisting that $\sigma^{a} \Sigma_{a}=\sigma^{a} \tilde{\Sigma}_{a}$. This can always be arranged by a change of gauge:

$$
\sigma^{a}\left(\Sigma_{a}-\tilde{\Sigma}_{a}\right) \mapsto \sigma^{a}\left(\Sigma_{a}-\tilde{\Sigma}_{a}\right)+2 \sigma^{a} E_{a} g
$$


and $\sigma^{a} E_{a}$ is a kind of Euler operator in $\sigma$, whereas $\sigma^{a}\left(\Sigma_{a}-\tilde{\Sigma}_{a}\right)$ as a power series in $\sigma$ has no zeroth order term. This choice of gauge was introduced independently by Harnad, Hurtubise, Légaré, and Shnider [11] as "transversal gauge". They use it to investigate Witten's constraint equations but do not consider the descent to $A_{(N)}$. This gauge fixing implies that further gauge freedom is confined to using $g$ which are independent of $\sigma$-i.e., ordinary holomorphic functions. These observations may be summarized as follows.

THEOREM. $H^{1}\left(A_{(N)}, 0\right)$ is isomorphic to the space of "potentials" $\Sigma_{a}(x, \sigma)$ and $\tilde{\Sigma}_{a}(x, \sigma)$, whose power series expansions in $\theta_{j}^{A}$ and $\tilde{\theta}^{A^{\prime} j}$ contain no irreducible components annihilated in forming $\tilde{\theta}^{A^{\prime} j} \Sigma_{A A^{\prime}}$ and $\theta_{j}^{A} \tilde{\Sigma}_{A A^{\prime}}$, respectively, and which satisfy

$$
\begin{aligned}
& \tilde{\theta}^{C^{\prime} j} \tilde{\theta}^{D^{\prime} k} \varepsilon_{C^{\prime} D^{\prime}}\left\{\delta_{\left(A^{\prime} \Sigma_{B) A^{\prime}}\right.}^{A^{\prime}}\right\}=0, \\
& \theta_{j}^{C} \theta_{k}^{D} \varepsilon_{C D}\left\{\tilde{\delta}_{\left(A^{\prime}\right.}^{A}, \tilde{\Sigma}_{\left.B^{\prime}\right) A}\right\}=0, \\
& \theta_{k}^{B} \tilde{\theta}^{B^{\prime} j}\left\{\delta_{A B^{\prime}} \tilde{\Sigma}_{B A^{\prime}}-\tilde{\delta}_{B A^{\prime}} \Sigma_{A B^{\prime}}\right\}=2 \delta_{k}^{j} \Theta_{a} \text { for some } \Theta_{a}(x, \sigma), \\
& \sigma^{a} \Sigma_{a}=\sigma^{a} \tilde{\Sigma}_{a}
\end{aligned}
$$

modulo the gauge freedom of $\Sigma_{a} \mapsto \Sigma_{a}+\delta_{a} g$ and $\tilde{\Sigma}_{a} \mapsto \tilde{\Sigma}_{a}+\tilde{\delta}_{a} g$ for an arbitrary function $g=g(x)$.

Notice that $\Theta_{a}$ is gauge invariant, as are the terms in the power series expansions of $\Sigma_{a}$ and $\tilde{\Sigma}_{a}$ above order zero. In some sense $\Sigma$ and $\tilde{\Sigma}$ may be thought of as self-dual and anti-self-dual parts, but note that it is not necessarily the case that $\delta_{(A}^{A^{\prime} \Sigma_{B) A^{\prime}}}$, for example, vanishes.

5. The Yang-Mills equations. One can now attempt systematically to find the general solution of the equations in the previous theorem. This is a rather tedious task carried out in $\S 7$ but the results are remarkably simple:

THEOREM. The general solution of the equations of the previous theorem are as follows. Only $\Sigma_{a}$ and $\tilde{\Sigma}_{a}$ need be given since $\Theta_{a}$ is determined by one of the equations.

$\underline{N=1}$ :

$$
\begin{aligned}
& \Sigma_{a}=\phi_{a}+\sigma^{b} \varepsilon_{A^{\prime} B^{\prime}}\left(p_{A B}+h \varepsilon_{A B}\right), \\
& \tilde{\Sigma}_{a}=\phi_{a}+\sigma^{b} \varepsilon_{A B}\left(p_{A^{\prime} B^{\prime}}+h \varepsilon_{A^{\prime} B^{\prime}}\right),
\end{aligned}
$$

where $\phi_{a}(x)$ and $h(x)$ are arbitrary,

$$
p_{A B}=-\frac{1}{2} D_{(A}^{A^{\prime}} \phi_{B) A^{\prime}}, \quad p_{A^{\prime} B^{\prime}}=\frac{1}{2} D_{\left(A^{\prime}\right.}^{A} \phi_{\left.B^{\prime}\right) A},
$$

and $\phi_{a}$ is determined up to the gauge freedom $\phi_{a} \mapsto \phi_{a}+D_{a} g$.

$\underline{N=2}$ :

$$
\begin{aligned}
\Sigma_{a}= & \phi_{a}+\sigma^{b}\left\{p_{A B} \varepsilon_{A^{\prime} B^{\prime}}+q_{A^{\prime} B^{\prime}} \varepsilon_{A B}\right\} \\
& +\sigma^{b} \sigma^{c}\left\{r_{A B C C^{\prime}} \varepsilon_{A^{\prime} B^{\prime}}+s_{C C^{\prime}} \varepsilon_{A B} \varepsilon_{A^{\prime} B^{\prime}}+t_{A A^{\prime}} \varepsilon_{B C} \varepsilon_{B^{\prime} C^{\prime}}\right\} \\
& +\sigma^{b} \sigma^{b} \sigma^{d}\left\{u_{A B} \varepsilon_{C D^{\prime}} \varepsilon_{A^{\prime} B^{\prime}} \varepsilon_{C^{\prime} D^{\prime}}\right\}, \\
\tilde{\Sigma}_{a}= & \phi_{a}+\sigma^{b}\left\{\tilde{p}_{A^{\prime} B^{\prime}} \varepsilon_{A B}+\tilde{q}_{A B} \varepsilon_{A^{\prime} B^{\prime}}\right\} \\
& +\sigma^{b} \sigma^{c}\left\{\tilde{r}_{A^{\prime} B^{\prime} C^{\prime} C} \varepsilon_{A B}+s_{C C^{\prime}} \varepsilon_{A B} \varepsilon_{A^{\prime} B^{\prime}}+t_{A A^{\prime}} \varepsilon_{B C} \varepsilon_{B^{\prime} C^{\prime}}\right\} \\
& +\sigma^{b} \sigma^{c} \sigma^{d}\left\{\tilde{u}_{A^{\prime} B^{\prime}} \varepsilon_{C^{\prime} D^{\prime}} \varepsilon_{A B} \varepsilon_{C D}\right\}
\end{aligned}
$$


where $\phi_{a}(x)$ is arbitrary,

$$
\begin{array}{ll}
p_{A B}=-\frac{1}{2} D_{(A}^{A^{\prime}} \phi_{B) A^{\prime}}, & \tilde{p}_{A^{\prime} B^{\prime}}=\frac{1}{2} D_{\left(A^{\prime}\right.}^{A} \phi_{\left.B^{\prime}\right) A}, \\
q_{A^{\prime} B^{\prime}}=3 \tilde{p}_{A^{\prime} B^{\prime}}, & \tilde{q}_{A B}=3 p_{A B}, \\
r_{A B C A^{\prime}}=-\frac{1}{3} D_{A^{\prime}(A} p_{B C)}, & \tilde{r}_{A^{\prime} B^{\prime} C^{\prime} A}=\frac{1}{3} D_{A\left(A^{\prime}\right.} \tilde{p}_{\left.B^{\prime} C^{\prime}\right)}, \\
s_{a}=\frac{14}{9} J_{a} & \text { where } J_{A A^{\prime}}=D_{A^{\prime}}^{B} p_{A B}\left(=-D_{A}^{\left.B^{\prime} \tilde{p}_{A^{\prime} B^{\prime}}\right)}\right. \\
t_{a}=-\frac{8}{9} J_{a}, & \\
u_{A B}=\frac{1}{6} D_{(A}^{A^{\prime}} J_{B) A^{\prime}}, & \tilde{u}_{A^{\prime} B^{\prime}}=-\frac{1}{6} D_{\left(A^{\prime}\right.}^{A} J_{\left.B^{\prime}\right) A},
\end{array}
$$

and $\phi_{a}$ is determined up to gauge freedom $\phi_{a} \mapsto \phi_{a}+D_{a} g$.

$\underline{N \geq 3}$

$$
\begin{aligned}
\Sigma_{a}= & \phi_{a}+\sigma^{b}\left\{-F_{b a}^{-}+3 F_{b a}^{+}\right\}+\sigma^{b} \sigma^{c}\left\{\frac{1}{3} D_{(c} F_{b) a}^{-}+\frac{5}{3} D_{(c} F_{b) a}^{+}\right\}+\cdots \\
= & \frac{2 \overbrace{\sigma^{b} \sigma^{c} \cdots \sigma^{e}}^{n}}{(n+1) !}\left\{(-1)^{n} D_{(e} \cdots D_{c} F_{b) a}^{-}+(2 n+1) D_{(e} \cdots D_{c} F_{b) a}^{+}\right\}+\cdots, \\
\tilde{\Sigma}_{a}= & \phi_{a}+\sigma^{b}\left\{F_{b a}^{+}-3 F_{b a}^{-}\right\}+\sigma^{b} \sigma^{c}\left\{\frac{1}{3} D_{(c} F_{b) a}^{+}+\frac{5}{3} D_{(c} F_{b) a}^{-}\right\}+\cdots \\
& +\frac{2 \overbrace{\sigma^{b} \sigma^{c} \cdots \sigma^{e}}^{n}}{(n+1) !}\left\{D_{(e} \cdots D_{c} F_{b) a}^{+}+(-1)^{n}(2 n+1) D_{(e} \cdots D_{c} F_{b) a}^{-}\right\}+\cdots,
\end{aligned}
$$

where $F_{a b}^{-}=-\frac{1}{2} D_{\left(A^{\prime}\right.}^{C^{\prime}} \phi_{B) C^{\prime}} \varepsilon_{A^{\prime} B^{\prime}}, F_{a b}^{+}=\frac{1}{2} D_{\left(A^{\prime}\right.}^{C} \phi_{\left.B^{\prime}\right) C} \varepsilon_{A B}$, and $D_{[a} F_{b c]}^{+}=0$ (or equivalently, $\left.D_{[a} F_{b c]}^{-}=0\right)$. In the notation from the case $N=2, F_{a b}^{-}=p_{A B} \varepsilon_{A^{\prime} B^{\prime}}$, $f_{a b}^{+}=p_{A^{\prime} B^{\prime}} \varepsilon_{A B^{\prime}}$ and $J_{a}=0$. In case $N \geq 3 \Phi_{a}$ is restricted by the equation $J_{a}=0$ (equivalently, $\square \phi_{a}=2 D_{A}^{B^{\prime}} D_{A^{\prime}}^{B} \phi_{b}$ for $\square=D^{b} D_{b}$ ) but is otherwise free and determined up to gauge freedom $\phi_{a} \mapsto \phi_{a}+D_{a} g$.

For this theorem and its corollary below there are complications for the nonAbelian case. For $N=1,2$, and 3 the obvious generalization holds for the nonAbelian case, but to extend a vector bundle from $A_{(3)}$ to $A_{(4)}$ there are genuine obstructions (see [14]) which show up on $M$. Although the supersymmetric method will prove this, the explicit computations rapidly get out of hand.

COROLlaRY. Including the case $N=0$ for completeness, $H^{a}\left(A_{(N)}, 0\right)$ has the following interpretation on $M$. Letting $G$ denote the space of holomorphic potentials/gauge on $M, H^{1}(A, 0) \cong G$. There is an exact sequence

$$
\begin{gathered}
0 \rightarrow\left\{h \varepsilon_{A B} \varepsilon_{A^{\prime} B^{\prime}}\right\} \rightarrow H^{1}\left(A_{(1)}, 0\right) \rightarrow G \rightarrow 0 . \\
H^{1}\left(A_{(2)}, 0\right) \cong G .
\end{gathered}
$$

For $N \geq 3, H^{1}\left(A_{(N)}, 0\right) \cong$ holomorphic source-free Maxwell fields on $M$ i.e. for which the current $J$ vanishes.

There are alternative methods of proving some of these statements using similar supersymmetric means. For example, one can prove $H^{1}\left(A_{(2)}, 0\right) \cong G$ as follows. From the exact sequence of sheaves on $A$,

$$
0 \rightarrow O_{(1)}(-1,-1) \rightarrow O_{(2)} \rightarrow 0 \rightarrow 0
$$


it follows that $H^{1}\left(A_{(2)}, 0\right) \cong H^{1}(A, 0) \cong G$ if and only if $H^{1}\left(A_{(1)}, O(-1,-1)\right)=0$. To show this consider the exact sequence

$$
0 \rightarrow O(-2,-2) \rightarrow O_{(1)}(-1,-1) \rightarrow O(-1,-1) \rightarrow 0 .
$$

By the Penrose transform [5] $H^{1}(A, O(-2,-2))=0$, so $H^{1}\left(A_{(1)}, \mathcal{O}(-1,-1)\right) \rightarrow$ $H^{1}(A, \mathcal{O}(-1,-1))$ is injective. This map factors through $H^{1}\left(A_{[1]}, \mathcal{O}(-1,-1)\right)$ so it suffices to show that $H^{1}\left(A_{[1]}, O(-1,-1)\right)=0$. If $F_{\alpha \beta}(Z, W, \zeta, \psi)$ is a representative cocycle then, forming $f_{\alpha \beta}$ from the superambitwistor incidence relations,

$$
\eta^{A} \partial_{A} f_{\alpha \beta}=0, \quad \pi^{A^{\prime}} \tilde{\delta}_{A^{\prime}} f_{\alpha \beta}=0, \quad \eta^{A} \pi^{A^{\prime}} D_{A A^{\prime}} f_{\alpha \beta}=0
$$

and splitting $f_{\alpha \beta}=f_{\alpha}-f_{\beta}$ gives $\eta^{A} \partial_{A} f_{\alpha}, \pi^{A^{\prime}} \tilde{\delta}_{A^{\prime}} f_{\alpha}$, and $\eta^{A} \pi^{A^{\prime}} D_{A A^{\prime}} f_{\alpha}$ globally defined as in an earlier argument. However, in this case $\eta^{A} \partial_{A} f_{\alpha}$ is homogeneous of degree $(-1,0)$ so necessarily vanishes. Similarly, $\pi^{A^{\prime}} \tilde{\delta}_{A^{\prime}} f_{\alpha}=0$ and, although $\eta^{A} \pi^{A^{\prime}} D_{A A^{\prime}} f_{\alpha}$ is homogeneous of degree zero, it vanishes too because

$$
2 \eta^{A} \pi^{A^{\prime}} D_{A A^{\prime}} f_{\alpha}=\eta^{A} \partial_{A}\left(\pi^{A^{\prime}} \tilde{\delta}_{A^{\prime}} f_{\alpha}\right)+\pi^{A^{\prime}} \tilde{\partial}_{A^{\prime}}\left(\eta^{A} \partial_{A} f_{\alpha}\right)
$$

These supersymmetric methods apply to calculations with other homogeneities also. For example $H^{1}\left(A_{(2)}, \mathcal{O}(-1,0)\right)$ is isomorphic to solutions of the neutrino equation on $M$ (as observed by Henkin and Manin [12]).

6. Formalities and rigor. Delaying for the moment the formal definition of a global supermanifold, the local calculus on a holomorphic supermanifold is as follows. Let $x$ be a local variable in $\mathbb{C}^{m}$. A superfunction is a finite formal sum

$$
F(x, y)=f(x)=y^{\alpha} f_{\alpha}(x)+\cdots+\overbrace{y^{\alpha} y^{\beta} \cdots y^{\delta}}^{n} f_{\alpha \beta \cdots \delta}(x)
$$

where the indices take values in $1,2, \ldots, n$ and the coefficients are skew in these indices. In other words, $F$ is a section of $\bigwedge^{*}\left(O^{n}\right)$. "Calculus" in the anticommuting variables is an entirely algebraic procedure. It is analogous with the calculus of formal power series (in commuting variables) and, indeed, the results are of the form of algebraic identities which all have counterparts in the commuting variables case obtained by interchange of symmetrization with antisymmetrization (reflection of Young tableau). In this sense it is possible, as observed by Roger Penrose, to regard supersymmetric calculus as negative dimensional ordinary tensor calculus [15]. As an example, consider the de Rham sequence in the category of formal power series:

$$
F(x)=f+x^{a} f_{a}+x^{a} x^{b} f_{a b}+\cdots
$$

for $a=1,2, \ldots, m$. The coefficients are symmetric in the indices. A $p$-form

$$
\Omega(x)=\Omega_{\underbrace{a \cdots c}_{p}}(x) d x^{a} \wedge \cdots \wedge d x^{c}
$$

for $\Omega_{a \cdots c}=\Omega_{[a \cdots c]}$ may also be expanded as a power series

$$
\Omega_{a \cdots c}(x)=\omega_{a \ldots c}+x^{d} \omega_{a \cdots c d}+x^{d} x^{e} \omega_{a \cdots c d e}+\cdots .
$$

Each coefficient in this expansion is skew-symmetric in the first $p$ indices and symmetric in the remainder. The $k$ th order term in such an expansion therefore has 
symmetries (using Young Tableau (see e.g. $[\mathbf{1 8}, \mathbf{2 5}]$ )).

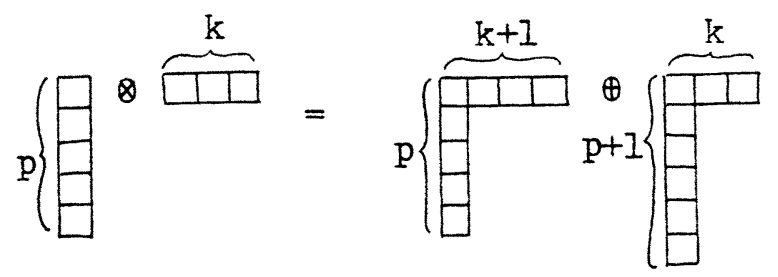

The entire de Rham sequence therefore takes the form

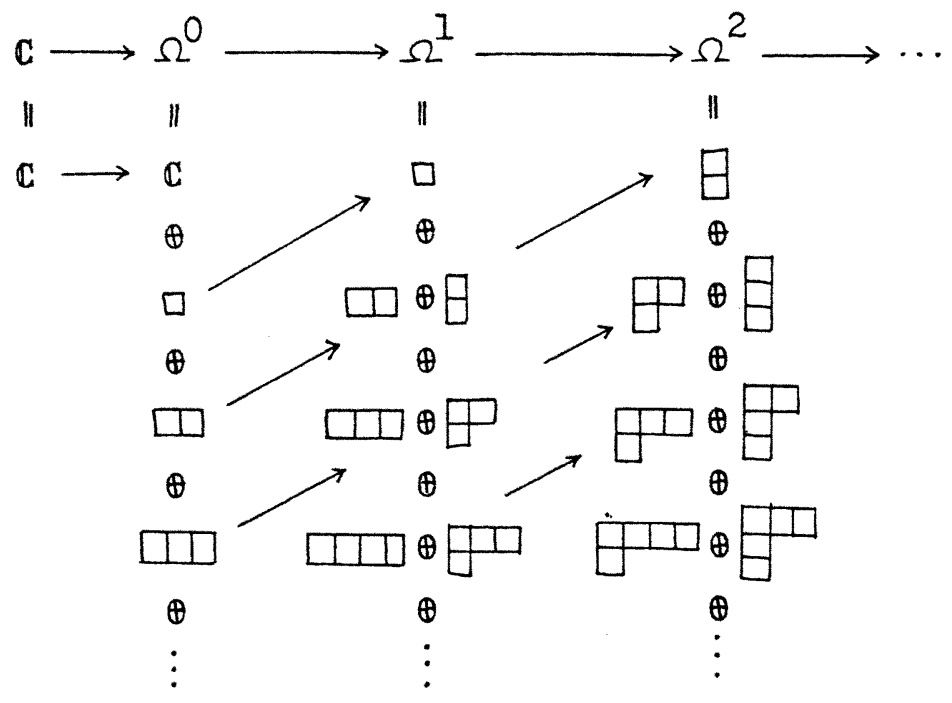

from which exactness (i.e. the Poincare lemma) is clear. The de Rham sequence in anticommuting variables has $p$-forms

$$
\Omega(y)=\Omega_{\underbrace{\alpha \cdots \gamma}_{p}}(y) d y^{\alpha} \odot \cdots \odot d y^{\gamma}
$$

for $\Omega_{\alpha \cdots \gamma}=\Omega_{(\alpha \cdots \gamma)}$ and, by definition, each coefficient is a formal series

$$
\Omega_{\alpha \cdots \gamma}(y)=\omega_{\alpha \cdots \gamma}+y^{\delta} \omega_{\alpha \cdots \gamma \delta}+y^{\delta} y^{\varepsilon} \omega_{\alpha \cdots \gamma \delta \varepsilon}+\cdots
$$

Each coefficient in this expansion is symmetric in the first $p$ indices and skew in the remainder. The $k$ th order term in such an expansion therefore has symmetries

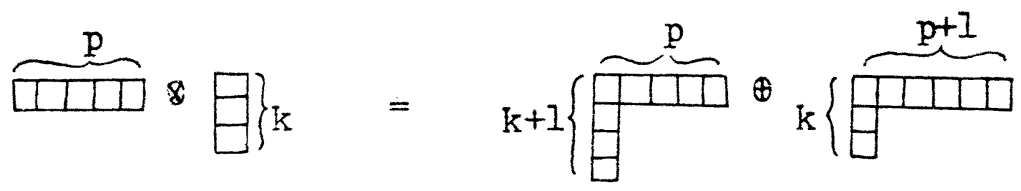


The entire de Rham sequence therefore takes the form

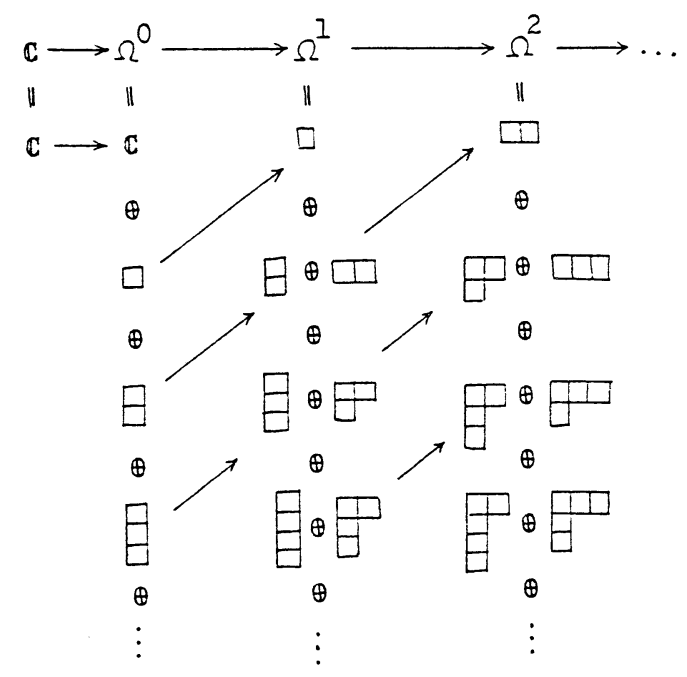

and again exactness is clear. The full de Rham sequence on a supermanifold is locally the tensor product of the ordinary de Rham sequence for the underlying manifold with the above construction for the anticommuting variables. There are similar comments for results such as the Frobenius integrability theorem.

Following Kostant [13], a supermanifold in the holomorphic category consists of an underlying classical holomorphic manifold $Q$ together with a sheaf of $\mathbb{Z}_{2}$ graded $\mathbb{C}$ algebras $O_{[n]}$ called the sheaf of germs of superfunctions. Locally $O_{[n]}$ is required to be of the form $\bigwedge^{\circ}\left(O^{n}\right)$ graded according to odd and even degree. $O_{[n]}$ is also required to be augmented over $O_{\text {; }}$ i.e., there is a $\mathbb{C}$ homomorphism $O_{[n]} \rightarrow 0$. Locally this augmentation is taking the degree zero part of $\bigwedge^{0}\left(O^{n}\right)$. This definition compares well with the precise idea of formal thickening given in the context of thickambitwistors in $\S 3$. Thickening $A$ is also achieved by augmenting the underlying classical sheaf. For both supersymmetric extensions and formal thickenings there is an interesting difference between the holomorphic and smooth categories. For the case of supermanifolds, Batchelor [1] has shown that in the smooth category the sheaf of superfunctions is always isomorphic to the sheaf of sections of $\bigwedge^{\circ} E$ for some smooth vector bundle $E$. In other words, the structure of a supermanifold contains no more information than that of an additional vector bundle. In the holomorphic case, however, there are nontrivial supermanifolds with structure over and above the underlying vector bundle. As pointed out by Green [10], this further information is contained in various holomorphic cohomology groups. The corresponding analysis for formal thickenings is carried out in [6]. Both $A_{(N)}$ and $A_{[N]}$ are nontrivial in this sense. They may be constructed as follows.

On PT $\times \mathrm{PT}^{*}$ the canonical section $\chi=Z^{\alpha} W_{\alpha}$ of $O(1,1)$ defines $A$ as its zero set and so allows one to identity $I=O(-1,-1)$ where $I$ is the ideal sheaf of $A$. Thus, the definition of $A_{(N)}$ given in $\S 3$ may be rewritten as an exact sequence on $\mathrm{PT} \times \mathrm{PT}^{*}$,

$$
O(-N-1,-N-1) \stackrel{\chi^{N+1}}{\rightarrow} O \rightarrow O_{(N)} \rightarrow 0
$$

noting that $O_{(N)}$ is supported on $A$. 
To define $A_{[M]}$ one can adopt an analogous procedure. First define $P_{[N]}$ as $P$ together with the structure sheaf $\bigwedge^{\bullet} O_{j}(-1)$. Thus, $P_{[N]}$ is the trivial supermanifold with underlying vector bundle $O_{j}(-1)$. This should better be written as $V \otimes_{\mathfrak{C}} O(-1)$ for $V$ an $N$-dimensional complex vector space since a particular basis for $V$ is unnecessary. In other words, the index $j$ is an abstract index $[\mathbf{1 8}]$. Up to isomorphism, there is no choice for this superstructure on $P$ since the relevant cohomology groups describing the freedom in choosing a superstructure based on $\bigwedge^{\cdot} O_{j}(-1)$ may be computed (by using the Bott-Borel-Weil theorem as explained for example in [5]) and turn out to be zero. Similarly, $P_{[N]}^{*}$ is defined to be $P^{*}$ with structure sheaf $\bigwedge^{\cdot} O^{j}(-1)=\bigwedge^{*}\left(V^{*} \otimes_{C} O(-1)\right)$. The supermanifold $P_{[N]} \times P_{[N]}^{*}$ is $P \times P^{*}$ with structure sheaf $S=\bigwedge^{*}\left(O_{j}(-1) \oplus O^{k}(-1)\right)$. A superfunction on $P_{[N]}$ may be written as a formal expansion

$$
F(Z, \zeta)=f(Z)+\varsigma^{j} f_{j}(Z)+\varsigma^{j} \varsigma^{k} f_{j k}(Z)+\cdots
$$

where

$$
\overbrace{i j \cdots k}^{n} \in O_{[i j \cdots k]}(-1)
$$

With a similar convention for $P_{[N]}^{*}, Z^{\alpha} W_{\alpha}-2 \zeta^{j} \psi_{j}$ is a canonically defined section of $S(1,1) \equiv O(1,1) \otimes_{\mathcal{O}} S$. This allows one to define $O_{[N]}$ supported on $A$ by means of the exact sequence

$$
S(-1,-1) \stackrel{Z^{\alpha} W_{\alpha}-2 \zeta^{j} \psi_{j}}{\rightarrow} S \rightarrow O_{[N]} \rightarrow 0 .
$$

This is tantamount to decreeing that $Z^{\alpha} W_{\alpha}=2 \zeta^{j} \psi_{j}$ in $O_{[N]}$. It is easy to show that $A_{[N]}$ comes equipped with a mapping $A_{[N]} \rightarrow A_{(N)}$ or, equivalently, a homomorphism of augmented rings $O_{(N)} \rightarrow O_{[N]}$.

More generally, a mapping between supermanifolds is defined to be a holomorphic mapping of the underlying classical manifolds together with a homomorphism of structure sheaves as described for general ringed spaces for example in $[\mathbf{2}, \mathbf{8}]$. It is in this sense that the incidence relations of $\S 3$ should be interpreted-i.e., as a correspondence (cf. $[\mathbf{3}, \mathbf{4}, \mathbf{5}]$ )

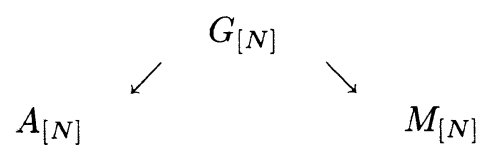

augmenting the classical correspondence between ambitwistor space and Minkowski space $[\mathbf{3}, \mathbf{5}]$.

It remains to make sense of $M_{[N]}$ and $G_{[N]}$. This section closes with a definition of $M_{[N]}$, leaving $G_{[N]}$ and other details to the reader. All these definitions follow the same theme. I would like to thank Toby Bailey for his critical appraisal of these constructions.

On $M \times M$ adopt the temporary convention that $O_{A^{\prime}}$ denotes the primed spin bundle on the first factor whereas $O_{A}$ should denote the unprimed spin bundle with respect to the second factor. Thus, $O_{A^{\prime}}$ and $O_{A}$ as vector bundles have fibres $K$ and $(T / L)^{*}$ over a point $(K, L) \in \mathrm{Gr}_{2}(T) \times \mathrm{Gr}_{2}(T)=M \times M$ (see [4]). Equip $M \times M$ with a superstructure sheaf

$$
S=\bigwedge^{\cdot}\left(O_{A^{\prime} j} \oplus O_{A}^{j}\right)=\bigwedge^{\cdot}\left(V \otimes O_{A^{\prime}} \oplus V^{*} \otimes O_{A}\right)
$$


Thus

$$
S=O \oplus\left[O_{A^{\prime} j} \oplus O_{A}^{j}\right] \oplus\left[O_{a} \oplus \cdots\right] \oplus \cdots
$$

and

$$
S^{a}=\mathcal{O}^{a} \otimes_{0} S=\mathcal{O}^{a} \oplus\left[O_{B^{\prime} j}^{a} \oplus \mathcal{O}_{B}^{a j}\right] \oplus\left[O_{b}^{a} \oplus \cdots\right] \oplus \cdots .
$$

Consider the bundle $\mathcal{O}^{a}$ on $M \times M$. Over $(K, L)$ it has fiber

$$
\dot{K}^{*} \otimes(T / L)=\operatorname{Hom}(K, T / L)
$$

and hence has a canonical section given by the composition $K \hookrightarrow T \rightarrow T / L$. Denote this section by $z^{a}$ and observe that it defines the diagonal $\{K=L\}$ inside $M \times M$. This diagonal is canonically isomorphic to $M$. The superstructure on $M$ is obtained by slightly modifying this defining function. Let $\sigma^{a}$ denote the section of $S^{a}$ given by the Kronecker delta $\delta_{b}^{a} \in O_{b}^{a}$ as in the above expansion of $S^{a}$. Formally, $\sigma^{a}=\theta^{A^{\prime} j} \theta_{j}^{A}$. Define $\mathcal{O}_{[N]}$ on $M$ by the exact sequence

$$
S_{a} \stackrel{z^{a} \rightarrow \sigma^{a}}{\rightarrow} S \rightarrow O_{[N]} \rightarrow 0 .
$$

Notice that $O_{[N]}$ is not $\mathbb{Z}$-graded since it is defined by a $\mathbb{Z}_{2}$-homogeneous but not Z-homogeneous ideal.

7. Appendix. It is the purpose of this appendix to prove the theorem of $\S 5$ in detail. This is a good example of spinor calculations in anticommuting variables.

Case $N=1$. Since $\Sigma_{a}$ must contain no terms annihilated by forming $\tilde{\theta}^{A^{\prime}} \Sigma_{A A^{\prime}}$ it follows that it must have the form

$$
\Sigma_{a}=\phi_{a}+\sigma^{b} \varepsilon_{A^{\prime} B^{\prime}} f_{A B} .
$$

Further decomposing $f_{A B}$ into its symmetric and skew parts, applying similar reasoning for $\tilde{\Sigma}_{a}$, and insisting that $\sigma^{a} \Sigma_{a}=\sigma^{a} \tilde{\Sigma}_{a}$ means that

$$
\begin{aligned}
& \Sigma_{a}=\phi_{a}+\sigma^{b} \varepsilon_{A^{\prime} B^{\prime}}\left(p_{A B}+h \varepsilon_{A B}\right), \\
& \tilde{\Sigma}_{a}=\phi_{a}+\sigma^{b} \varepsilon_{A B}\left(\tilde{p}_{A^{\prime} B^{\prime}}+h \varepsilon_{A^{\prime} B^{\prime}}\right),
\end{aligned}
$$

and we are left with the equations

$$
\tilde{\theta}^{C^{\prime}} \tilde{\theta}^{D^{\prime}} \varepsilon_{C^{\prime} D^{\prime}}\left\{\delta_{(A}^{A^{\prime} \Sigma_{B) A^{\prime}}}\right\}=0
$$

and

$$
\begin{aligned}
& \theta^{C} \theta^{D} \varepsilon_{C D}\left\{\tilde{\delta}_{\left(A^{\prime}\right.}^{A}, \tilde{\Sigma}_{\left.B^{\prime}\right) A}\right\}=0 . \\
& \delta_{\left(A^{\prime} \Sigma_{B) A^{\prime}}\right.}^{A^{\prime}}=D_{(A}^{A^{\prime} \phi_{B) A^{\prime}}}+\varepsilon_{A^{\prime}}{A^{\prime}}_{p_{A B}}+\sigma^{c} \varepsilon_{A^{\prime} C^{\prime}}\left(D_{(A}^{A^{\prime}} p_{B) C}+D_{(A}^{A^{\prime}} h \varepsilon_{B) C}\right) \\
& =D_{(A}^{A^{\prime}} \phi_{B) A^{\prime}}+2 p_{A B}+\sigma^{c}\left(D_{C^{\prime}(A} P_{B) C}+D_{(A}^{A^{\prime} h \varepsilon_{B) C}}\right) \\
& \Rightarrow \tilde{\theta}^{C^{\prime}} \tilde{\theta}^{D^{\prime}} \varepsilon_{C^{\prime} D^{\prime}}\left\{\delta_{(A}^{A^{\prime} \Sigma_{B) A^{\prime}}}\right\}=\tilde{\theta}^{C^{\prime}} \tilde{\theta}^{D^{\prime}} \varepsilon_{C^{\prime} D^{\prime}}\left(D_{(A}^{A^{\prime}} \phi_{B) A^{\prime}}+2 p_{A B}\right) \text {. }
\end{aligned}
$$

Thus, $p_{A B}=-\frac{1}{2} D_{\left(A^{\prime}\right.}^{A^{\prime}} \phi_{B) A^{\prime}}$ and similarly $p_{A^{\prime} B^{\prime}}=\frac{1}{2} D_{\left(A^{\prime}\right.}^{A} \phi_{\left.B^{\prime}\right) A^{\prime}}$.

Case $N=2$. Here and more generally it is perhaps not quite so obvious what symmetries are imposed on the coefficients of an expansion in $\sigma$ by virtue of the anticommuting nature of $\theta_{j}^{A}$ and $\tilde{\theta}^{A^{\prime} j}$. These symmetries may be expressed in terms of Young tableau [25]. 
For example

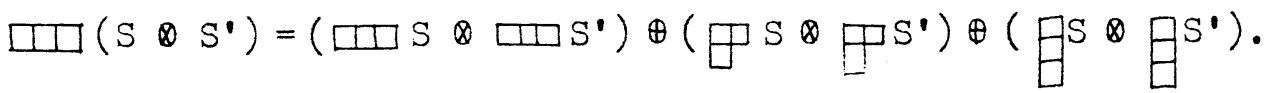

For spinors, $S$ and $S^{\prime}$ are 2-dimensional so the last term vanishes. If $N=2$ and we decompose $\chi_{b c d}$ in $\sigma^{b} \sigma^{c} \sigma^{d} \chi_{b c d}$ according to this formula then only the term in

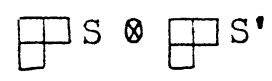

survives because $\theta_{i}^{(B} \theta_{j}^{C} \theta_{k}^{D)}=0$. Therefore the term in $\sigma^{b} \sigma^{c} \sigma^{d}$, for example, in the expansion of $\Sigma_{a}$,

$$
\Sigma_{a}=\cdots+\sigma^{b} \sigma^{c} \sigma^{d} \chi_{a b c d}+\cdots,
$$

has symmetries and hence spinor decomposition of the form

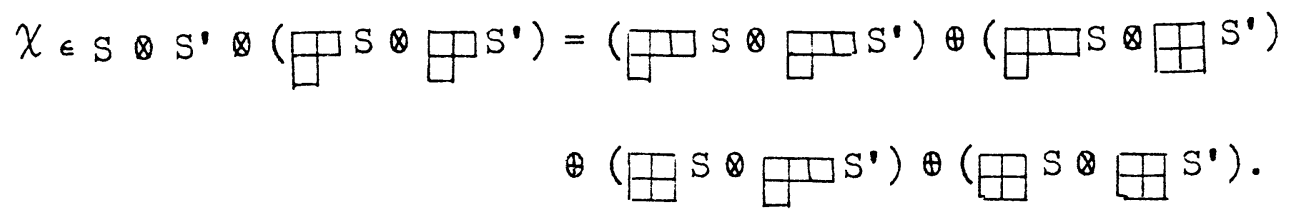

However, in forming $\tilde{\theta}^{A^{\prime}} \Sigma_{A A^{\prime}}$ some of the representations are annihilated so, finally, the term in $\sigma^{b} \sigma^{c} \sigma^{d}$ may be taken to have the form

$$
\Sigma_{a}=\cdots+\sigma^{b} \sigma^{c} \sigma^{d}\left\{u_{A B} \varepsilon_{C D^{D}} \varepsilon_{A^{\prime} B^{\prime}} \varepsilon_{C^{\prime} D^{\prime}}+v \varepsilon_{A B} \varepsilon_{C D^{\prime}} \varepsilon_{A^{\prime} B^{\prime}} \varepsilon_{C^{\prime} D^{\prime}}\right\}+\cdots
$$

where $u_{A B}(x)$ is symmetric in the indices $A$ and $B$. Similar reasoning applied to other terms and to $\tilde{\Sigma}_{a}$ and insisting that $\sigma^{a} \Sigma_{a}=\sigma^{a} \tilde{\Sigma}_{a}$ means that $\Sigma_{a}$ and $\tilde{\Sigma}_{a}$ take the form

$$
\begin{aligned}
& \Sigma_{a}=\phi_{a}+\sigma^{b} G_{a b}+\sigma^{b} \sigma^{c}\left\{r_{A B C C^{\prime}} \varepsilon_{A^{\prime} B^{\prime}}+s_{C C^{\prime}} \varepsilon_{A B^{B}} \varepsilon_{A^{\prime} B^{\prime}}+t_{A A^{\prime}} \varepsilon_{B C} \varepsilon_{B^{\prime} C^{\prime}}\right\} \\
& +\sigma^{b} \sigma^{c} \sigma^{d}\left\{u_{A B} \varepsilon_{C D} \varepsilon_{A^{\prime} B^{\prime}} \varepsilon_{C^{\prime} D^{\prime}}+v \varepsilon_{A B} \varepsilon_{C D} \varepsilon_{A^{\prime} B^{\prime}} \varepsilon_{C^{\prime} D^{\prime}}\right\}, \\
& \tilde{\Sigma}_{a}=\phi_{a}+\sigma^{b} \tilde{G}_{a b}+\sigma^{b} \sigma^{c}\left\{\tilde{r}_{A^{\prime} B^{\prime} C^{\prime} C} \varepsilon_{A B}+\tilde{s}_{C C^{\prime}} \varepsilon_{A B} \varepsilon_{A^{\prime} B^{\prime}}+\tilde{t}_{A A^{\prime}} \varepsilon_{B C} \varepsilon_{B^{\prime} C^{\prime}}\right\} \\
& +\sigma^{b} \sigma^{c} \sigma^{d}\left\{\tilde{u}_{A^{\prime} B^{\prime}} \varepsilon_{C^{\prime} D^{\prime}} \varepsilon_{A B} \varepsilon_{C D}+v \varepsilon_{A B} \varepsilon_{C D} \varepsilon_{A^{\prime} B^{\prime}} \varepsilon_{C^{\prime} D^{\prime}}\right\}
\end{aligned}
$$

where $G_{(a b)}=\tilde{G}_{(a b)}$ and $s_{a}+t_{a}=\tilde{s}_{a}+\tilde{t}_{a}$.

It remains to impose the differential equations

$$
\begin{aligned}
& \tilde{\theta}^{C^{\prime} j} \tilde{\theta}^{D^{\prime} k} \varepsilon_{C^{\prime} D^{\prime}}\left\{\delta_{\left(A^{A^{\prime}} \Sigma_{B) A^{\prime}}\right.}^{A^{\prime}}=0=\theta_{j}^{C} \theta_{k}^{D} \varepsilon_{C D}\left\{\tilde{\delta}_{\left(A^{\prime}\right.}^{A} \tilde{\Sigma}_{\left.B^{\prime}\right) A}\right\},\right. \\
& \theta_{k}^{B} \tilde{\theta}^{B^{\prime} j}\left\{\delta_{A B^{\prime}} \tilde{\Sigma}_{B A^{\prime}}-\tilde{\delta}_{B A^{\prime}} \Sigma_{A B^{\prime}}\right\}=2 \delta_{k}^{j} \Theta_{a} \text { for some } \Theta_{a}(x, \sigma) \text {. }
\end{aligned}
$$


To study these equations it is necessary to prove the following purely algebraic lemmata (which assume $N=2$ ).

LEMMA 1. $\tilde{\theta}^{C^{\prime} j} \tilde{\theta}^{D^{\prime} k} \varepsilon_{C^{\prime} D^{\prime}}\{\chi(x, \sigma)\}=0$ if and only if $\chi$ is of the form

$$
\chi=\sigma^{p} \sigma^{q} \alpha_{p q}+\text { higher order terms }
$$

where $\varepsilon^{P Q} \varepsilon^{P^{\prime} Q^{\prime}} \alpha_{p q}=0$ (or, equivalently, $\left.\alpha_{p q}=\alpha_{(P Q)\left(P^{\prime} Q^{\prime}\right)}\right)$.

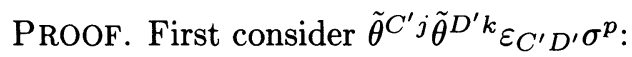

$$
\tilde{\theta}^{C^{\prime} 1} \tilde{\theta}^{D^{\prime} 1} \varepsilon_{C^{\prime} D^{\prime}}\left(\theta_{1}^{P} \tilde{\theta}^{P^{\prime} 1}+\theta_{2}^{P} \tilde{\theta}^{P^{\prime} 2}\right)=\tilde{\theta}^{C^{\prime} 1} \tilde{\theta}^{D^{\prime} 1} \varepsilon_{C^{\prime} D^{\prime}} \theta_{2}^{P} \tilde{\theta}^{P^{\prime} 2}
$$

so already the term in $\sigma^{p}$ in the expansion of $\chi$ must vanish. Next consider $\tilde{\theta} C^{\prime} j \tilde{\theta}^{D^{\prime} k} \varepsilon_{C^{\prime} D^{\prime}} \sigma^{p} \sigma^{a}$ :

$$
\begin{aligned}
& \tilde{\theta}^{C^{\prime} 1} \tilde{\theta}^{D^{\prime} 1} \varepsilon_{C^{\prime} D^{\prime}}\left(\theta_{1}^{P} \tilde{\theta}^{P^{\prime} 1}+\theta_{2}^{P} \tilde{\theta}^{P^{\prime} 2}\right)\left(\theta_{1}^{Q} \tilde{\theta}^{Q^{\prime} 1}+\theta_{2}^{Q} \tilde{\theta}^{Q^{\prime} 2}\right) \\
& =\frac{1}{4}\left[\tilde{\theta}^{C^{\prime} 1} \tilde{\theta}_{C^{\prime}}^{1}\right]\left[\theta_{2}^{R} \theta_{R 2}\right]\left[\tilde{\theta}^{R^{\prime} 2} \tilde{\theta}_{R^{\prime}}^{2}\right] \varepsilon^{P Q} \varepsilon^{P^{\prime} Q^{\prime}}, \\
& \tilde{\theta}^{C^{\prime} 1} \tilde{\theta}^{D^{\prime 2} 2} \varepsilon_{C^{\prime} D^{\prime}}\left(\theta_{1}^{P} \tilde{\theta}^{P^{\prime} 1}+\theta_{2}^{P} \tilde{\theta}^{P^{\prime 2}}\right)\left(\theta_{1}^{Q} \tilde{\theta}^{Q^{\prime} 1}+\theta_{2}^{Q} \tilde{\theta}^{Q^{\prime 2}}\right) \\
& =\tilde{\theta}^{C^{\prime} 1} \tilde{\theta}^{P^{\prime} 1} \varepsilon_{C^{\prime} D^{\prime}} \tilde{\theta}^{D^{\prime} 2} \tilde{\theta}^{Q^{\prime} 2} \theta_{1}^{P} \theta_{2}^{Q}+\tilde{\theta}^{C^{\prime} 1} \tilde{\theta}^{Q^{\prime} 1} \varepsilon_{C^{\prime} D^{\prime}} \tilde{\theta}^{D^{\prime} 2} \tilde{\theta}^{P^{\prime} 2} \theta_{1}^{Q} \theta_{2}^{P} \\
& =\frac{1}{4}\left[\tilde{\theta}^{R^{\prime} 1} \tilde{\theta}_{R^{\prime}}^{1}\right]\left[\tilde{\theta}^{S^{\prime} 2} \tilde{\theta}_{S^{\prime}}^{2}\right]\left(\varepsilon^{C^{\prime} P^{\prime}} \varepsilon_{C^{\prime} D^{\prime}} \varepsilon^{D^{\prime} Q^{\prime}} \theta_{1}^{P} \theta_{2}^{Q}+\varepsilon^{C^{\prime} Q^{\prime}} \varepsilon_{C^{\prime} D^{\prime}} \varepsilon^{D^{\prime} P^{\prime}} \theta_{1}^{Q} \theta_{2}^{P}\right) \\
& =\frac{1}{4}\left[\tilde{\theta}^{R^{\prime} 1} \tilde{\theta}_{R^{\prime}}^{1}\right]\left[\tilde{\theta}^{S^{\prime} 2} \tilde{\theta}_{S^{\prime}}^{2}\right]\left(\theta_{1}^{P} \theta_{2}^{Q} \varepsilon^{P^{\prime} Q^{\prime}}-\theta_{1}^{Q} \theta_{2}^{P} \varepsilon^{P^{\prime} Q^{\prime}}\right) \\
& =-\frac{1}{4}\left[\tilde{\theta}^{R^{\prime} 1} \tilde{\theta}_{R^{\prime}}^{1}\right]\left[\tilde{\theta}^{S^{\prime} 2} \tilde{\theta}_{S^{\prime}}^{2}\right]\left[\theta_{1}^{R} \theta_{R 2}\right] \varepsilon^{P G} \varepsilon^{P^{\prime} Q^{\prime}} \text {. }
\end{aligned}
$$

Similarly, $\tilde{\theta}^{C^{\prime} 2} \tilde{\theta}^{D^{\prime} 1} \varepsilon_{C^{\prime} D^{\prime}} \sigma^{p} \sigma^{q}$ and $\tilde{\theta}^{C^{\prime} 2} \tilde{\theta}^{D^{\prime} 2} \varepsilon_{C^{\prime} D^{\prime}} \sigma^{p} \sigma^{q}$ are proportional to $\varepsilon^{P Q} \varepsilon^{P^{\prime} Q^{\prime}}$. Hence $\varepsilon^{P Q} \varepsilon^{P^{\prime} Q^{\prime}} \alpha_{p q}=0$, but otherwise there are no conditions on $\alpha_{p q}$. All higher

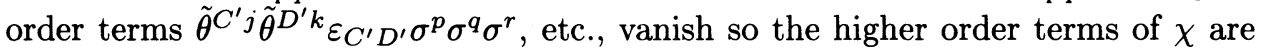
unrestricted.

LEMMA 2. In terms of an expansion in $\sigma$

$$
\chi_{b}(x, \sigma)=\alpha_{b}+\sigma^{p} \alpha_{b p}+\sigma^{p} \sigma^{q} \alpha_{b p q}+\sigma^{p} \sigma^{q} \sigma^{r} \alpha_{b p q r}+\cdots,
$$

$\theta_{k}^{B} \tilde{\theta}^{B^{\prime} j}\left\{\chi_{b}(x, \sigma)\right\}=2 \delta_{k}^{j} \Theta$ for some $\Theta(x, \sigma)$ if and only if $\alpha_{b}=0, \varepsilon^{B P} \alpha_{b p}=0$, $\varepsilon^{B^{\prime} P^{\prime}} \alpha_{b p}=0$, and $\varepsilon^{P Q} \varepsilon^{P^{\prime} Q^{\prime}}\left[2 \alpha_{p b q}-\alpha_{b p q}\right]=0$.

ProOF. Consider $\theta_{k}^{B} \tilde{\theta}^{B^{\prime} j} \sigma^{p}$ for $j=1,2$ and $k=1,2$ :

$$
\begin{aligned}
\theta_{1}^{B} \tilde{\theta}^{B^{\prime} 2} \sigma^{p} & =\theta_{1}^{B} \tilde{\theta}^{B^{\prime} 2}\left(\theta_{1}^{P} \tilde{\theta}^{P^{\prime} 1}+\theta_{2}^{P} \tilde{\theta}^{P^{\prime} 2}\right) \\
& =\frac{1}{2}\left[\theta_{1}^{Q} \theta_{Q 1}\right] \varepsilon^{B P} \tilde{\theta}^{B^{\prime} 2} \tilde{\theta}^{P^{\prime} 1}+\frac{1}{2}\left[\tilde{\theta}^{Q^{\prime} 2} \tilde{\theta}_{Q^{\prime}}^{2}\right] \varepsilon^{B^{\prime} P^{\prime}} \theta_{1}^{B} \theta_{2}^{P}, \\
\theta_{2}^{B} \tilde{\theta}^{B^{\prime} 1} \sigma^{p} & =\theta_{2}^{B} \tilde{\theta}^{B^{\prime} 1}\left(\theta_{1}^{P} \tilde{\theta}^{P^{\prime} 1}+\theta_{2}^{P} \tilde{\theta}^{P^{\prime} 2}\right) \\
& =\frac{1}{2}\left[\tilde{\theta}^{Q^{\prime} 1} \tilde{\theta}_{Q^{\prime}}^{1}\right] \varepsilon^{B^{\prime} P^{\prime}} \theta_{2}^{B} \theta_{1}^{P}+\frac{1}{2}\left[\theta_{2}^{Q} \theta_{Q 2}\right] \varepsilon^{B P} \tilde{\theta}^{B^{\prime} 1} \tilde{\theta}^{P^{\prime} 2}, \\
\theta_{1}^{B} \tilde{\theta}^{B^{\prime} 1} \sigma^{p} & =\theta_{1}^{B} \tilde{\theta}^{B^{\prime} 1}\left(\theta_{1}^{P} \tilde{\theta}^{P^{\prime} 1}+\theta_{2}^{P} \tilde{\theta}^{P^{\prime} 2}\right) \\
& =-\frac{1}{4}\left[\theta_{1}^{Q} \theta_{Q 1}\right]\left[\tilde{\theta}^{Q^{\prime} 1} \tilde{\theta}_{Q^{\prime}}^{1}\right] \varepsilon^{B P} \varepsilon^{B^{\prime} P^{\prime}}+\theta_{1}^{B} \tilde{\theta}^{B^{\prime} 1} \theta_{2}^{P} \tilde{\theta}^{P^{\prime} 2}, \\
\theta_{2}^{B} \tilde{\theta}^{B^{\prime} 2} \sigma^{p} & =\theta_{2}^{B} \tilde{\theta}^{B^{\prime} 2}\left(\theta_{1}^{P} \tilde{\theta}^{P^{\prime} 1}+\theta_{2}^{P} \tilde{\theta}^{P^{\prime} 2}\right) \\
& =-\frac{1}{4}\left[\theta_{2}^{Q} \theta_{Q 2}\right]\left[\tilde{\theta}^{Q^{\prime} 2} \tilde{\theta}_{Q^{\prime}}^{2}\right] \varepsilon^{B P} \varepsilon^{B^{\prime} P^{\prime}}+\theta_{2}^{B} \tilde{\theta}^{B^{\prime} 2} \theta_{1}^{P} \tilde{\theta}^{P^{\prime} 1} .
\end{aligned}
$$


Now $\varepsilon^{B P} \alpha_{b p}=0=\varepsilon^{B^{\prime} P^{\prime}} \alpha_{b p} \Leftrightarrow \alpha_{B P B^{\prime} Q^{\prime}}=\alpha_{(B P)\left(B^{\prime} Q^{\prime}\right)}$, in which case

$$
\theta_{j}^{B} \tilde{\theta}^{B^{\prime} k} \sigma^{p} \alpha_{b p}=0 \text { if } j \neq k
$$

and

$$
\begin{aligned}
\alpha_{1}^{B} \tilde{\theta}^{B^{\prime} 1} \sigma^{p} \alpha_{b p} & =\theta_{1}^{B} \tilde{\theta}^{B^{\prime} 1} \theta_{2}^{P} \tilde{\theta}^{P^{\prime} 2} \alpha_{B P B^{\prime} P^{\prime}}=\theta_{1}^{P} \tilde{\theta}^{P^{\prime} 1} \theta_{2}^{B} \tilde{\theta}^{B^{\prime} 2} \alpha_{B P B^{\prime} P^{\prime}} \\
& =\theta_{2}^{B} \tilde{\theta}^{B^{\prime} 2} \theta_{1}^{P} \tilde{\theta}^{P^{\prime} 1} \alpha_{B P B^{\prime} P^{\prime}}=\theta_{2}^{B} \tilde{\theta}^{B^{\prime} 2} \sigma^{b} \alpha_{b p}
\end{aligned}
$$

as required.

Next consider $\theta_{k}^{B} \tilde{\theta}^{B^{\prime} j} \sigma^{p} \sigma^{q}$ :

$$
\begin{aligned}
\theta_{1}^{B} \tilde{\theta}^{B^{\prime} 2} \sigma^{p} \sigma^{q}= & \theta_{1}^{B} \tilde{\theta}^{B^{\prime} 2}\left(\theta_{1}^{P} \tilde{\theta}^{P^{\prime} 1}+\theta_{2}^{P} \tilde{\theta}^{P^{\prime} 2}\right)\left(\theta_{1}^{Q} \tilde{\theta}^{Q^{\prime} 1}+\theta_{2}^{Q} \tilde{\theta}^{Q^{\prime} 2}\right) \\
= & \frac{1}{4}\left[\theta_{1}^{R} \theta_{R 1}\right]\left[\tilde{\theta}^{R^{\prime} 2} \tilde{\theta}_{R^{\prime}}^{2}\right]\left(\theta_{2}^{Q} \tilde{\theta}^{P^{\prime} 1} \varepsilon^{B P} \varepsilon^{B^{\prime} Q^{\prime}}+\theta_{2}^{P} \tilde{\theta}^{Q^{\prime} 1} \varepsilon^{B Q} \varepsilon^{B^{\prime} P^{\prime}}\right), \\
\theta_{2}^{B} \tilde{\theta}^{B^{\prime} 1} \sigma^{p} \sigma^{q}= & \frac{1}{4}\left[\theta_{2}^{R} \theta_{R 2}\right]\left[\tilde{\theta}^{R^{\prime} 1} \tilde{\theta}_{R^{\prime}}^{1}\right]\left(\theta_{1}^{P} \tilde{\theta}^{Q^{\prime} 2} \varepsilon^{B Q} \varepsilon^{B^{\prime} P^{\prime}}+\theta_{1}^{Q} \tilde{\theta}^{P^{\prime} 2} \varepsilon^{B P} \varepsilon^{B^{\prime} Q^{\prime}}\right), \\
\theta_{1}^{B} \tilde{\theta}^{B^{\prime} 1} \sigma^{p} \sigma^{q}= & \theta_{1}^{B} \tilde{\theta}^{B^{\prime} 1}\left(\theta_{1}^{P} \tilde{\theta}^{P^{\prime} 1}+\theta_{2}^{P} \tilde{\theta}^{P^{\prime} 2}\right)\left(\theta_{1}^{Q} \tilde{\theta}^{Q^{\prime} 1}+\theta_{2}^{Q} \tilde{\theta}^{Q^{\prime} 2}\right) \\
= & \theta_{1}^{B} \tilde{\theta}^{B^{\prime} 1} \theta_{1}^{P} \tilde{\theta}^{P^{\prime} 1} \theta_{2}^{Q} \tilde{\theta}^{Q^{\prime} 2}+\theta_{1}^{B} \tilde{\theta}^{B^{\prime} 1} \theta_{2}^{P} \tilde{\theta}^{P^{\prime} 2} \theta_{1}^{Q} \tilde{\theta}^{Q^{\prime} 1} \\
& +\theta_{1}^{B} \tilde{\theta}^{B^{\prime} 1} \theta_{2}^{P} \tilde{\theta}^{P^{\prime} 2} \theta_{2}^{Q} \tilde{\theta}^{Q^{\prime} 2} \\
= & -\frac{1}{4}\left[\theta_{1}^{R} \theta_{R 1}\right]\left[\tilde{\theta}^{R^{\prime} 1} \tilde{\theta}_{R^{\prime}}^{1}\right]\left(\varepsilon^{B P} \varepsilon^{B^{\prime} P^{\prime}} \theta_{2}^{Q} \tilde{\theta}^{Q^{\prime} 2}+\varepsilon^{B Q} \varepsilon^{B^{\prime} Q^{\prime}} \theta_{2}^{P} \tilde{\theta}^{P^{\prime} 2}\right) \\
& -\frac{1}{4}\left[\theta_{2}^{R} \theta_{R 2}\right]\left[\tilde{\theta}^{R^{\prime} 2} \tilde{\theta}_{R^{\prime}}^{2}\right]\left(\varepsilon^{P Q} \varepsilon^{P^{\prime} Q^{\prime}} \theta_{1}^{B} \tilde{\theta}^{B^{\prime} 1}\right), \\
\theta_{2}^{B} \tilde{\theta}^{B^{\prime} 2} \sigma^{p} \sigma^{q}= & -\frac{1}{4}\left[\theta_{2}^{R} \theta_{R 2}\right]\left[\tilde{\theta}^{R^{\prime} 2} \tilde{\theta}_{R^{\prime}}^{2}\right]\left(\varepsilon^{B Q} \varepsilon^{B^{\prime} Q^{\prime}} \theta^{P} \tilde{\theta}_{1}^{P^{\prime} 1}+\varepsilon^{B P} \varepsilon^{B^{\prime} P^{\prime}} \theta_{1}^{Q} \tilde{\theta}^{Q^{\prime} 1}\right) \\
& -\frac{1}{4}\left[\theta_{1}^{R} \theta_{R 1}\right]\left[\tilde{\theta}^{R^{\prime} 1} \tilde{\theta}_{R^{\prime}}^{1}\right]\left(\varepsilon^{P Q} \varepsilon^{P^{\prime} Q^{\prime}} \theta_{2}^{B} \tilde{\theta}^{B^{\prime} 2}\right) .
\end{aligned}
$$

Since $\alpha_{b p q}$ is symmetric in $p$ and $q$, it admits an irreducible spinor decomposition:

$$
\begin{aligned}
\alpha_{b p q}= & \alpha_{B B^{\prime}} \varepsilon_{P Q} \varepsilon_{P^{\prime} Q^{\prime}}+\alpha_{B P Q B^{\prime} P^{\prime} Q^{\prime}}+\alpha_{B P Q\left(P^{\prime}\right.} \varepsilon_{\left.Q^{\prime}\right) B^{\prime}} \\
& +\varepsilon_{B(P} \alpha_{Q) B^{\prime} P^{\prime} Q^{\prime}}+\varepsilon_{B(P} \beta_{Q)\left(P^{\prime}\right.} \varepsilon_{\left.Q^{\prime}\right) B^{\prime}}
\end{aligned}
$$

where each spinor field on the right-hand side is symmetric in its indices. Thus,

$$
\begin{aligned}
\theta_{1}^{B} \tilde{\theta}^{B^{\prime} 2} \sigma^{p} \sigma^{q} \alpha_{b p q}= & \frac{1}{4}\left[\theta_{1}^{R} \theta_{R 1}\right]\left[\tilde{\theta}^{R^{\prime} 2} \tilde{\theta}_{R^{\prime}}^{2}\right]\left(-2 \theta_{2}^{S} \tilde{\theta}^{S^{\prime} 1} \alpha_{S S^{\prime}}-\frac{9}{2} \theta_{2}^{S} \tilde{\theta}^{S^{\prime} 1} \beta_{S S^{\prime}}\right), \\
\theta_{2}^{B} \tilde{\theta}^{B^{\prime} 1} \sigma^{p} \sigma^{q} \alpha_{b p q}= & \frac{1}{4}\left[\theta_{2}^{R} \theta_{R 2}\right]\left[\tilde{\theta}^{R^{\prime} 1} \tilde{\theta}_{R^{\prime}}^{1}\right]\left(-2 \theta_{1}^{S} \tilde{\theta}^{S^{\prime} 2} \alpha_{S S^{\prime}}-\frac{9}{2} \theta_{1}^{S} \tilde{\theta}^{S^{\prime} 2} \beta_{S S^{\prime}}\right), \\
\theta_{1}^{B} \tilde{\theta}^{B^{\prime} 1} \sigma^{p} \sigma^{q} \alpha_{b p q}= & -\frac{1}{4}\left[\theta_{1}^{R} \theta_{R 1}\right]\left[\tilde{\theta}^{R^{\prime} 1} \tilde{\theta}_{R^{\prime}}^{1}\right]\left(2 \theta_{2}^{S} \tilde{\theta}^{S^{\prime} 2} \alpha_{S S^{\prime}}-\frac{9}{2} \theta_{2}^{S} \tilde{\theta}^{S^{\prime} 2} \beta_{S S^{\prime}}\right) \\
& -\frac{1}{4}\left[\theta_{2}^{R} \theta_{R 2}\right]\left[\tilde{\theta}^{R^{\prime} 2} \tilde{\theta}_{R^{\prime}}^{2}\right]\left(4 \theta_{1}^{S} \theta^{S^{\prime} 1} \alpha_{S S^{\prime}}\right), \\
\theta_{2}^{B} \tilde{\theta}^{B^{\prime} 2} \sigma^{p} \sigma^{q} \alpha_{b p q}= & -\frac{1}{4}\left[\theta_{2}^{R} \theta_{R 2}\right]\left[\tilde{\theta}^{R^{\prime} 2} \tilde{\theta}_{R^{\prime}}^{2}\right]\left(2 \theta_{1}^{S} \tilde{\theta}^{S^{\prime} 1} \alpha_{S S^{\prime}}-\frac{9}{2} \theta_{1}^{S} \tilde{\theta}^{S^{\prime} 1} \beta_{S S^{\prime}}\right) \\
& -\frac{1}{4}\left[\theta_{1}^{R} \theta_{R 1}\right]\left[\tilde{\theta}^{R^{\prime} 1} \tilde{\theta}_{R^{\prime}}^{1}\right]\left(4 \theta_{2}^{S} \tilde{\theta}^{S^{\prime} 2} \alpha_{S S^{\prime}}\right) .
\end{aligned}
$$

So $\theta_{k}^{B} \tilde{\theta}^{B^{\prime} j} \sigma^{p} \sigma^{q} \alpha_{b p q}=2 \delta_{k}^{j} \Gamma$ if and only if $4 \alpha_{s}+9 \beta_{s}=0$. But

$$
\varepsilon^{P Q} \varepsilon^{P^{\prime} Q^{\prime}} \alpha_{b p q}=4 \alpha_{b} \text { and } \varepsilon^{P Q} \varepsilon^{P^{\prime} Q^{\prime}} \alpha_{p b q}=\alpha_{b}-\frac{9}{4} \beta_{b}
$$

so

$$
4 \alpha_{s}+9 \beta_{s}=\varepsilon^{P Q} \varepsilon^{P^{\prime} Q^{\prime}}\left[-4 \alpha_{p b q}+2 \alpha_{b p q}\right] .
$$

Hence $\theta_{k}^{B} \tilde{\theta}^{B^{\prime} j} \sigma^{p} \sigma^{q} \alpha_{b p q}=2 \delta_{k}^{j} \Gamma$ if and only if $\varepsilon^{P Q} \varepsilon^{P^{\prime} Q^{\prime}}\left[2 \alpha_{b p q}-\alpha_{b p q}\right]=0$. Higher order terms are easily seen to impose no extra conditions. 
Recall that $\Sigma_{a}=\phi_{a}+\sigma^{b} G_{a b}+\cdots$ and $\tilde{\Sigma}_{a}=\phi_{a}+\sigma^{b} \tilde{G}_{a b}+\cdots$ where $G_{(a b)}=\tilde{G}_{(a b)}$. Applying Lemma 2 to

$$
\delta_{A B^{\prime}} \tilde{\Sigma}_{B A^{\prime}}-\tilde{\delta}_{B A^{\prime}} \Sigma_{A B^{\prime}}=D_{A B^{\prime}} \phi_{B A^{\prime}}+\tilde{G}_{B A^{\prime} A B^{\prime}}-D_{B A^{\prime}} \phi_{A B^{\prime}}+G_{A B^{\prime} B A^{\prime}}+\cdots,
$$

it follows that $G_{a b}$ and $\tilde{G}_{a b}$ are skew. Thus they may be written

$$
G_{a b}=p_{A B} \varepsilon_{A^{\prime} B^{\prime}}+q_{A^{\prime} B^{\prime}} \varepsilon_{A B}, \quad \tilde{G}_{a b}=\tilde{p}_{A^{\prime} B^{\prime}} \varepsilon_{A B}+\tilde{q}_{A B} \varepsilon_{A^{\prime} B^{\prime}},
$$

where $p, q, \tilde{p}$, and $\tilde{q}$ are symmetric in their indices. Hence, Lemma 2 implies

$$
0=D_{A B^{\prime}} \phi_{B A^{\prime}}+\tilde{p}_{A^{\prime} B^{\prime}} \varepsilon_{B A}+\tilde{q}_{A B} \varepsilon_{A^{\prime} B^{\prime}}-D_{B A^{\prime}} \phi_{A B^{\prime}}+p_{A B} \varepsilon_{B^{\prime} A^{\prime}}+q_{A^{\prime} B^{\prime}} \varepsilon_{A B},
$$

which, by contracting with $\varepsilon^{A B}$ and $\varepsilon^{A^{\prime} B^{\prime}}$, is equivalent to

$$
0=-2 D_{\left(A^{\prime}\right.}^{A} \phi_{\left.B^{\prime}\right) A}-2 \tilde{p}_{A^{\prime} B^{\prime}}+2 q_{A^{\prime} B^{\prime}}, \quad 0=2 D_{(A}^{A^{\prime}} \phi_{B) A^{\prime}}+2 \tilde{q}_{A B}-2 p_{A B} .
$$

Also, by applying Lemma 1 to $\delta_{(A}^{A^{\prime} \Sigma_{B) A^{\prime}}}=D_{(A}^{A} \phi_{B) B^{\prime}}+2 p_{A B}+\cdots$,

$$
0=D_{(A}^{A^{\prime}} \phi_{B) A^{\prime}}+2 p_{A B},
$$

and a similar argument for $\tilde{\delta}_{\left(A^{\prime}\right.}^{A} \tilde{\Sigma}_{\left.B^{\prime}\right) A}$ implies

$$
0=D_{\left(A^{\prime}\right.}^{A} \phi_{\left.B^{\prime}\right) A}-2 \tilde{p}_{A^{\prime} B^{\prime}}
$$

These equations have the unique solution

$$
\begin{array}{ll}
p_{A B}=-\frac{1}{2} D_{(A}^{A^{\prime}} \phi_{B) A^{\prime}}, & \tilde{p}_{A^{\prime} B^{\prime}}=\frac{1}{2} D_{\left(A^{\prime}\right.}^{A} \phi_{\left.B^{\prime}\right) A}, \\
q_{A^{\prime} B^{\prime}}=3 \tilde{p}_{A^{\prime} B^{\prime}}, & \tilde{q}_{A B}=3 p_{A B} .
\end{array}
$$

Hence $p$ and $q$ are determined in the expansion of $\Sigma$ :

$$
\begin{aligned}
& \Sigma_{a}=\phi_{a}+\sigma^{b}\left\{p_{A B} \varepsilon_{A^{\prime} B^{\prime}}+q_{A^{\prime} B^{\prime}} \varepsilon_{A B}\right\} \\
& +\sigma^{b} \sigma^{c}\left\{r_{A B C C^{\prime}} \varepsilon_{A^{\prime} B^{\prime}}+s_{C C^{\prime}} \varepsilon_{A B} \varepsilon_{A^{\prime} B^{\prime}}+t_{A A^{\prime}} \varepsilon_{B C} \varepsilon_{B^{\prime} C^{\prime}}\right\} \\
& +\sigma^{b} \sigma^{c} \sigma^{d}\left\{u_{A B} \varepsilon_{C D} \varepsilon_{A^{\prime} B^{\prime}} \varepsilon_{C^{\prime} D^{\prime}}+v \varepsilon_{A B} \varepsilon_{C D} \varepsilon_{A^{\prime} B^{\prime}} \varepsilon_{C^{\prime} D^{\prime}}\right\} \\
& =\phi_{a}+\sigma^{b}\left\{p_{A B} \varepsilon_{A^{\prime} B^{\prime}}+q_{A^{\prime} B^{\prime}} \varepsilon_{A B}\right\} \\
& +\sigma^{b} \sigma^{c}\left\{\frac{1}{2} r_{A B C C^{\prime}} \varepsilon_{A^{\prime} B^{\prime}}+\frac{1}{2} r_{A B C B^{\prime}} \varepsilon_{A^{\prime} C^{\prime}}+\frac{1}{2} s_{C C^{\prime}} \varepsilon_{A B} \varepsilon_{A^{\prime} B^{\prime}}\right. \\
& \left.+\frac{1}{2} s_{B B^{\prime}} \varepsilon_{A C} \varepsilon_{A^{\prime} C^{\prime}}+t_{A A^{\prime}} \varepsilon_{B C} \varepsilon_{B^{\prime} C^{\prime}}\right\} \\
& +\sigma^{b} \sigma^{c} \sigma^{d}\left\{\frac{1}{3} u_{A B} \varepsilon_{C D^{2}} \varepsilon_{A^{\prime} B^{\prime}} \varepsilon_{C^{\prime} D^{\prime}}+\frac{1}{3} u_{A C} \varepsilon_{B D} \varepsilon_{A^{\prime} C^{\prime}} \varepsilon_{B^{\prime} D^{\prime}}\right. \\
& +\frac{1}{3} u_{A D} \varepsilon_{B C} \varepsilon_{A^{\prime} D^{\prime}} \varepsilon_{B^{\prime} C^{\prime}}+\frac{1}{3} v \varepsilon_{A B} \varepsilon_{C D^{\prime}} \varepsilon_{A^{\prime} B^{\prime}} \varepsilon_{C^{\prime} D^{\prime}}
\end{aligned}
$$

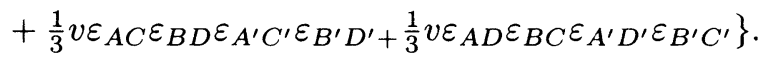

In the second version the coefficients have been arranged symmetric in $b c d \ldots$ which facilitates differentiation:

$$
\begin{aligned}
\delta_{(A}^{A^{\prime} \Sigma_{B) A^{\prime}}} & =D_{(A}^{A^{\prime}} \phi_{B) A^{\prime}}+2 p_{A B} \\
+ & \sigma^{c}\left\{D_{C^{\prime}(A} p_{B) C}-\varepsilon_{C(A} D_{B)}^{A^{\prime}} q_{A^{\prime} C^{\prime}}+3 r_{A B C C^{\prime}}-\varepsilon_{C(A} s_{B) C^{\prime}}+2 \varepsilon_{C(A} t_{B) C^{\prime}}\right\} \\
+ & \sigma^{c} \sigma^{d}\left\{\frac{1}{2} D_{C^{\prime}\left(A_{B}\right) C D D^{\prime}}+\frac{1}{2} D_{D^{\prime}(A} r_{B) C D C^{\prime}}-\frac{1}{2} \varepsilon_{C(A} D_{B) C^{\prime}} s_{D D^{\prime}}\right. \\
& \left.\quad-\frac{1}{2} \varepsilon_{D(A} D_{B) D^{\prime}} s_{C C^{\prime}}+D_{(A}^{A^{\prime}} t_{B) A^{\prime}} \varepsilon_{C D} \varepsilon_{C^{\prime} D^{\prime}}+3 u_{A B} \varepsilon_{C D} \varepsilon_{C^{\prime} D^{\prime}}\right\}
\end{aligned}
$$


Plugging this expression into Lemma 1 gives

$$
\begin{gathered}
D_{C^{\prime}\left({ }_{A}\right.} p_{B) C}-\varepsilon_{C(A} D_{B)}^{A^{\prime}} q_{A^{\prime} C^{\prime}}+3 r_{A B C C^{\prime}}-\varepsilon_{C\left(A_{A}\right.} s_{B) C^{\prime}}+2 \varepsilon_{C(A} t_{B) C^{\prime}}=0, \\
D_{\left(A_{B}{ }^{\prime} s_{B} A^{\prime}\right.}+4 D_{\left(A_{B} A^{\prime}\right.}^{A_{B} A^{\prime}}+12 u_{A B}=0 .
\end{gathered}
$$

The first of these equations decomposes (by contracting with $\varepsilon^{B C}$ ) into the two equations:

$-\frac{1}{2} D_{C^{\prime}}^{C} p_{A C}+3 D_{A}^{A^{\prime}} q_{A^{\prime} C^{\prime}}+\frac{3}{2} s_{A C^{\prime}}-3 t_{A C^{\prime}}=0 \quad$ and $\quad D_{C^{\prime}\left({ }_{A} p_{B C)}+3 r_{A B C C^{\prime}}=0 .\right.}$

This fixes $r$ as claimed in $\S 5$ and, letting $J_{a}=D_{A^{\prime}}^{B} p_{A B}\left(=-D_{A}^{B^{\prime}} \tilde{p}_{A^{\prime} B^{\prime}}\right)$, there remain the following equations (after a similar argument based on $\tilde{\delta}_{\left(A^{\prime}\right.}^{A} \tilde{\Sigma}_{\left.B^{\prime}\right) A}$ ):

$$
\begin{array}{ll}
-5 J_{a}+\frac{3}{2} s_{a}-3 t_{a}=0, & 5 J_{a}-\frac{3}{2} \tilde{s}_{a}+3 \tilde{t}_{a}=0, \\
D_{\left(A^{\prime} s_{B) A^{\prime}}+4 D_{(A}^{A^{\prime}} t_{B) A^{\prime}}+12 u_{A B}=0,\right.} & D_{\left(A^{\prime}\right.}^{A} \tilde{s}_{\left.B^{\prime}\right) A}+4 D_{\left(A^{\prime}\right.}^{A} \tilde{t}_{\left.B^{\prime}\right) A}=12 \tilde{u}_{A^{\prime} B^{\prime}}=0
\end{array}
$$

and (from earlier gauge fixing) $s_{a}+t_{a}=\tilde{s}_{a}+\tilde{t}_{a}$.

There are not quite enough equations here to fix $s, t, \tilde{s}, \tilde{t}, u$ and $\tilde{u}$.

Now consider the remaining differential equation:

$$
\begin{aligned}
& \delta_{A B^{\prime}} \tilde{\Sigma}_{B A^{\prime}}-\tilde{\delta}_{B A^{\prime}} \Sigma_{A B^{\prime}} \\
& =D_{A B^{\prime}} \phi_{B A^{\prime}}-D_{B A^{\prime}} \phi_{A B^{\prime}}-\tilde{p}_{A^{\prime} B^{\prime}} \varepsilon_{A B}+\tilde{q}_{A B} \varepsilon_{A^{\prime} B^{\prime}}-p_{A B} \varepsilon_{A^{\prime} B^{\prime}}+q_{A^{\prime} B^{\prime}} \varepsilon_{A B} \\
& +\sigma^{p}\left\{D_{A B^{\prime}} \tilde{p}_{A^{\prime} P^{\prime}} \varepsilon_{B P}+D_{A B^{\prime}} \tilde{q}_{B P} \varepsilon_{A^{\prime} P^{\prime}}-D_{B A^{\prime}} p_{A P} \varepsilon_{B^{\prime} P^{\prime}}-D_{B A^{\prime}} q_{B^{\prime} P^{\prime}} \varepsilon_{A P}\right. \\
& -\tilde{r}_{A^{\prime} B^{\prime} P^{\prime} P} \varepsilon_{A B}+\tilde{r}_{A^{\prime} B^{\prime} P^{\prime} A} \varepsilon_{B P}-\tilde{s}_{P P^{\prime}} \varepsilon_{A B} \varepsilon_{A^{\prime} B^{\prime}}+\tilde{s}_{A B^{\prime}} \varepsilon_{B P} \varepsilon_{A^{\prime} P^{\prime}} \\
& +2 \tilde{t}_{B A^{\prime}} \varepsilon_{A P} \varepsilon_{B^{\prime} P^{\prime}}-r_{A B P P^{\prime}} \varepsilon_{A^{\prime} B^{\prime}}+r_{A B P A^{\prime}} \varepsilon_{B^{\prime} P^{\prime}}-s_{P P^{\prime}} \varepsilon_{A B} \varepsilon_{A^{\prime} B^{\prime}} \\
& \left.+s_{B A^{\prime}} \varepsilon_{A P} \varepsilon_{B^{\prime} P^{\prime}}+2 t_{A B^{\prime}} \varepsilon_{B P} \varepsilon_{A P^{\prime}}\right\} \\
& +\sigma^{p} \sigma^{q}\left\{\frac{1}{2} D_{A B^{\prime}} \tilde{r}_{A^{\prime} P^{\prime} Q^{\prime} Q} \varepsilon_{B P}+\frac{1}{2} D_{A B^{\prime}} \tilde{r}_{A^{\prime} P^{\prime} Q^{\prime} P} \varepsilon_{B Q}\right. \\
& +\frac{1}{2} D_{A B^{\prime}} \tilde{s}_{Q Q^{\prime}} \varepsilon_{B P} \varepsilon_{A^{\prime} P^{\prime}}+\frac{1}{2} D_{A B^{\prime}} \tilde{s}_{P P^{\prime}} \varepsilon_{B Q} \varepsilon_{A^{\prime} Q^{\prime}} \\
& +D_{A B^{\prime}} \tilde{t}_{B A^{\prime}} \varepsilon_{P Q^{\prime}} \varepsilon_{P^{\prime} Q^{\prime}}-\frac{1}{2} D_{B A^{\prime}} r_{A P Q Q^{\prime}} \varepsilon_{B^{\prime} P^{\prime}} \\
& -\frac{1}{2} D_{B A^{\prime}} r_{A P Q P^{\prime}} \varepsilon_{B^{\prime} Q^{\prime}}-\frac{1}{2} D_{B A^{\prime}} s_{Q Q^{\prime}} \varepsilon_{A P} \varepsilon_{B^{\prime} P^{\prime}} \\
& -\frac{1}{2} D_{B A^{\prime}} s_{P P^{\prime}} \varepsilon_{A Q} \varepsilon_{B^{\prime} Q^{\prime}}-\frac{1}{2} D_{B A^{\prime}} t_{A B^{\prime}} \varepsilon_{P Q^{\prime}} \varepsilon_{P^{\prime} Q^{\prime}} \\
& -\tilde{u}_{A^{\prime} B^{\prime}} \varepsilon_{P^{\prime} Q^{\prime}} \varepsilon_{A B} \varepsilon_{P Q}+\tilde{u}_{A^{\prime} P^{\prime}} \varepsilon_{B^{\prime} Q^{\prime}} \varepsilon_{B P} \varepsilon_{A Q} \\
& +\tilde{u}_{A^{\prime} Q^{\prime}} \varepsilon_{B^{\prime} P^{\prime}} \varepsilon_{B Q} \varepsilon_{A P}-v \varepsilon_{A^{\prime} B^{\prime}} \varepsilon_{P^{\prime} Q^{\prime}} \varepsilon_{A B} \varepsilon_{P Q} \\
& +v \varepsilon_{A^{\prime} P^{\prime}} \varepsilon_{B^{\prime} Q^{\prime}} \varepsilon_{B P} \varepsilon_{A Q}+v \varepsilon_{A^{\prime} Q^{\prime}} \varepsilon_{B^{\prime} P^{\prime}} \varepsilon_{B Q} \varepsilon_{A P} \\
& -u_{A B} \varepsilon_{P Q} \varepsilon_{A^{\prime} B^{\prime}} \varepsilon_{P^{\prime} Q^{\prime}}+u_{A P} \varepsilon_{B Q^{\prime}} \varepsilon_{B^{\prime} P^{\prime}} \varepsilon_{A^{\prime} Q^{\prime}} \\
& +u_{A Q} \varepsilon_{B P} \varepsilon_{B^{\prime} Q^{\prime}} \varepsilon_{A^{\prime} P^{\prime}}-v \varepsilon_{A B} \varepsilon_{P Q} \varepsilon_{A^{\prime} B^{\prime}} \varepsilon_{P^{\prime} Q^{\prime}} \\
& \left.+v \varepsilon_{A P} \varepsilon_{B Q} \varepsilon_{B^{\prime} P^{\prime}} \varepsilon_{A^{\prime} Q^{\prime}}+v \varepsilon_{A Q} \varepsilon_{B P} \varepsilon_{B^{\prime} Q^{\prime}} \varepsilon_{A^{\prime} P^{\prime}}\right\} .
\end{aligned}
$$

Utilizing Lemma 2 on the coefficient of $\sigma^{p}$ gives the following new restrictions:

$$
\begin{aligned}
& 2 D_{A B^{\prime}} \tilde{p}_{A^{\prime} P^{\prime}}+D_{A^{\prime}}^{B} p_{A B} \varepsilon_{B^{\prime} P^{\prime}}-D_{A A^{\prime}} q_{B^{\prime} P^{\prime}}+3 \tilde{r}_{A^{\prime} B^{\prime} P^{\prime} A}+\tilde{s}_{A P^{\prime}} \varepsilon_{A^{\prime} B^{\prime}}+2 \tilde{s}_{A B^{\prime}} \varepsilon_{A^{\prime} P^{\prime}} \\
& +2 \tilde{t}_{A A^{\prime}} \varepsilon_{B^{\prime} P^{\prime}}+s_{A P^{\prime}} \varepsilon_{A^{\prime} B^{\prime}}+s_{A A^{\prime}} \varepsilon_{B^{\prime} P^{\prime}}+4 t_{A B^{\prime}} \varepsilon_{A^{\prime} P^{\prime}}=0, \\
& -D_{A}^{B^{\prime}} \tilde{p}_{A^{\prime} B^{\prime}} \varepsilon_{B P}+D_{A A^{\prime}} \tilde{q}_{B P}-2 D_{B A^{\prime}} p_{A P}+\tilde{s}_{P A^{\prime}} \varepsilon_{A B}+\tilde{s}_{A A^{\prime}} \varepsilon_{B P}+4 \tilde{t}_{B A^{\prime}} \varepsilon_{A P} \\
& +3 r_{A B P A^{\prime}}+s_{P A^{\prime}} \varepsilon_{A B}+2 s_{B A^{\prime}} \varepsilon_{A P}+2 t_{A A^{\prime}} \varepsilon_{B P}=0 .
\end{aligned}
$$


Now $f_{A B C}=0$ if and only if $f_{(A B C)}=0, \varepsilon^{A C} f_{A B C}=0$, and $\varepsilon^{B C} f_{A B C}=0$. Hence these two equations are equivalent to the following system of five:

$$
\begin{aligned}
& 2 D_{A\left(A^{\prime}\right.} \tilde{p}_{\left.B^{\prime} P^{\prime}\right)}-D_{A\left(A^{\prime}\right.} q_{\left.B^{\prime} P^{\prime}\right)}+3 \tilde{r}_{A^{\prime} B^{\prime} P^{\prime} A}=0 \text {, } \\
& D_{A^{\prime}(A} \tilde{q}_{B P)}-2 D_{A^{\prime}(A} p_{B P)}+3 r_{A B P A^{\prime}}=0 \text {, } \\
& D_{A^{\prime}}^{B} p_{A B}+D_{A}^{B^{\prime}} q_{A^{\prime} B^{\prime}}+5 \tilde{s}_{a}+2 \tilde{t}_{a}+2 s_{a}+8 t_{a}=0, \\
& -D_{A}^{B^{\prime}} \tilde{p}_{A^{\prime} B^{\prime}}-D_{A^{\prime}}^{B} \tilde{q}_{A B}+2 \tilde{s}_{a}+8 \tilde{t}_{a}+5 s_{a}+2 t_{a}=0 \text {, } \\
& -2 D_{A}^{B^{\prime}} \tilde{p}_{A^{\prime} B^{\prime}}+2 D_{A^{\prime}}^{B} p_{A B}+\tilde{s}_{a}+4 \tilde{t}_{a}+s_{a}+4 t_{a}=0 .
\end{aligned}
$$

The first two of these equations reduce to $\tilde{r}_{A^{\prime} B^{\prime} C^{\prime} A}=\frac{1}{3} D_{A\left(A^{\prime}\right.} \tilde{p}_{\left.B^{\prime} C^{\prime}\right)}$ and $r_{A B C A^{\prime}}=$ $-\frac{1}{3} D_{A^{\prime}(A} p_{B C)}$ so this is consistent with the same conclusion from earlier investigation of $\delta_{(A}^{A^{\prime} \Sigma_{B) A^{\prime}}}$ and $\tilde{\delta}_{\left(A^{\prime}\right.}^{A}, \tilde{\Sigma}_{\left.B^{\prime}\right) A}$. The remaining three equations, together with earlier restrictions reduce to

$$
\begin{array}{ll}
-2 J_{a}+5 \tilde{s}_{a}+2 \tilde{t}_{a}+2 s_{a}+8 t_{a}=0, & -2 J_{a}+2 \tilde{s}_{a}+8 \tilde{t}_{a}+5 s_{a}+2 t_{a}=0 \\
4 J_{a}+\tilde{s}_{a}+4 \tilde{t}_{a}+s_{a}+4 t_{a}=0, & s_{a}+t_{a}-\tilde{s}_{a}-\tilde{t}_{a}=0 \\
-5 J_{a}+\frac{3}{2} s_{a}-3 t_{a}=0, & 5 J_{a}-\frac{3}{2} \tilde{s}_{a}+3 \tilde{t}_{a}=0
\end{array}
$$

and, although overdetermined, these equations are consistent and have the unique solution $s_{a}=\tilde{s}_{a}=\frac{14}{9} J_{a}$ and $t_{a}=\tilde{t}_{a}=-\frac{8}{9} J_{a}$. Feeding these values into the previous equations for $u_{A B}$ and $u_{A^{\prime} B^{\prime}}$ gives $u_{A B}=\frac{1}{6} D_{(A}^{A^{\prime}} J_{B) A^{\prime}}$ and $u_{A^{\prime} B^{\prime}}=$ $-\frac{1}{6} D_{\left(A^{\prime}\right.}^{A} J_{\left.B^{\prime}\right) A}$ as claimed in $\S 5$. It remains to study the consequences of Lemma 2 for the coefficient of $\sigma^{p} \sigma^{q}$, namely

$$
\begin{aligned}
& 0= \varepsilon^{P Q} \varepsilon^{P^{\prime} Q^{\prime}}\left[2\left\{\frac{1}{2} D_{A P^{\prime}} \tilde{r}_{A^{\prime} B^{\prime} Q^{\prime} Q \varepsilon_{P B}}+\cdots\right\}-\left\{\frac{1}{2} D_{A B^{\prime}} r_{A^{\prime} P^{\prime} Q^{\prime}} \varepsilon_{B P}+\cdots\right\}\right] \\
&= 2\left\{-\frac{3}{2} D_{A}^{P^{\prime}} \tilde{r}_{A^{\prime} B^{\prime} P^{\prime} B}-\frac{1}{2} D_{A}^{P^{\prime}} \tilde{s}_{B P^{\prime}} \varepsilon_{A^{\prime} B^{\prime}}+D_{A A^{\prime}} \tilde{s}_{B B^{\prime}}+D_{A B^{\prime}} \tilde{t}_{B A^{\prime}}\right. \\
&+\frac{3}{2} D_{A^{\prime}}^{P} r_{A B P B^{\prime}}+\frac{1}{2} D_{A^{\prime}}^{P} s_{P B^{\prime}} \varepsilon_{A B}-D_{A A^{\prime}} s_{B B^{\prime}}-D_{B A^{\prime}} t_{A B^{\prime}} \\
&\left.+3 \tilde{u}_{A^{\prime} B^{\prime}} \varepsilon_{A B}+6 v \varepsilon_{A B} \varepsilon_{A^{\prime} B^{\prime}}+3 u_{A B} \varepsilon_{A^{\prime} B^{\prime}}\right\} \\
&-\left\{D_{A B^{\prime}} \tilde{s}_{B A^{\prime}}+4 D_{A B^{\prime}} \tilde{t}_{B A^{\prime}}-D_{B A^{\prime}} s_{A B^{\prime}}-4 D_{B A^{\prime}} t_{A B^{\prime}}\right. \\
&\left.-6 \tilde{u}_{A^{\prime} B^{\prime}} \varepsilon_{A B}-12 v \varepsilon_{A B} \varepsilon_{A^{\prime} B^{\prime}}-6 u_{A B} \varepsilon_{A^{\prime} B^{\prime}}\right\} .
\end{aligned}
$$

Substituting in this equation for $s, \tilde{s}, t, \tilde{t}, u$, and $\tilde{u}$ yields

$$
\begin{aligned}
3\left(D_{A}^{P^{\prime}} \tilde{r}_{A^{\prime} B^{\prime} P^{\prime} B}-D_{A^{\prime}}^{P} r_{A B P B^{\prime}}\right)= & \frac{4}{9} D_{A}^{P^{\prime}} J_{B P^{\prime}} \varepsilon_{A^{\prime} B^{\prime}}-\frac{4}{9} D_{A^{\prime}}^{P} J_{P B^{\prime}} \varepsilon_{A B} \\
& +\frac{2}{9}\left(D_{A B^{\prime}} J_{B A^{\prime}}-D_{B A^{\prime}} J_{A B^{\prime}}\right)+24 v \varepsilon_{A B} \varepsilon_{A^{\prime} B^{\prime}} .
\end{aligned}
$$

The left-hand side of this equation, however, may be rewritten

$$
\begin{aligned}
& 3\left(D_{A}^{P^{\prime}} \tilde{r}_{A^{\prime} B^{\prime} P^{\prime} B}-D_{A^{\prime}}^{P} r_{A B P B^{\prime}}\right)=D_{A}^{P^{\prime}} D_{B\left(A^{\prime}\right.} \tilde{p}_{\left.B^{\prime} P^{\prime}\right)}+D_{A^{\prime}}^{P} D_{B^{\prime}(A} p_{B P)} \\
& =\frac{1}{3}\left(D_{A}^{P^{\prime}} D_{B A^{\prime}} \tilde{p}_{B^{\prime} P^{\prime}}+D_{A}^{P^{\prime}} D_{B B^{\prime}} \tilde{p}_{A^{\prime} P^{\prime}}+D_{A}^{P^{\prime}} D_{B P^{\prime}} \tilde{p}_{A^{\prime} B^{\prime}}\right. \\
& \left.+D_{A^{\prime}}^{P} D_{B^{\prime} A} p_{B P}+D_{A^{\prime}}^{P} D_{B^{\prime} B} p_{A P}+D_{A^{\prime}}^{P} D_{B^{\prime} P} p_{A B}\right) \\
& =\frac{1}{3}\left(-D_{B A^{\prime}} J_{A B^{\prime}}-D_{B B^{\prime}} J_{A A^{\prime}}-\frac{1}{2} \varepsilon_{A B} \square \tilde{p}_{A^{\prime} B^{\prime}}\right. \\
& \left.+D_{A B^{\prime}} J_{B A^{\prime}}+D_{B B^{\prime}} J_{A A^{\prime}}-\frac{1}{2} \varepsilon_{A^{\prime} B^{\prime}} \square p_{A B}\right) \\
& =\frac{1}{3}\left(D_{A B^{\prime}} J_{B A^{\prime}}-D_{B A^{\prime}} J_{A B^{\prime}}\right)-\frac{1}{6}\left(\varepsilon_{A B} \square \tilde{p}_{A^{\prime} B^{\prime}}+\varepsilon_{A^{\prime} B^{\prime}} \square p_{A B}\right) \text {. }
\end{aligned}
$$


In particular, this expression is skew symmetric in $a$ and $b$. Thus, $v=0$ and by contracting both sides with $\varepsilon^{A B}$ and $\varepsilon^{A^{\prime} B^{\prime}}$ it remains to verify

$$
\begin{aligned}
& -\frac{2}{3} D_{A^{\prime}}^{A} J_{A B^{\prime}}-\frac{1}{3} \square \tilde{p}_{A^{\prime} B^{\prime}}=-\frac{8}{9} D_{A^{\prime}}^{A} J_{A B^{\prime}}-\frac{4}{9} D_{A^{\prime}}^{A} J_{A B^{\prime}}, \\
& \frac{2}{3} D_{A}^{A^{\prime}} J_{B A^{\prime}}-\frac{1}{3} \square p_{A B}=\frac{8}{9} D_{A}^{A^{\prime}} J_{B A^{\prime}}+\frac{4}{9} D_{A}^{A^{\prime}} J_{B A^{\prime}} .
\end{aligned}
$$

But $D_{A^{\prime}}^{A} J_{A B^{\prime}}=D_{A^{\prime}}^{A}\left(-D_{A}^{C^{\prime}} p_{B^{\prime} C^{\prime}}\right)=\frac{1}{2} \square p_{A^{\prime} B^{\prime}}$ and similarly, $D_{A}^{A^{\prime}} J_{B A^{\prime}}=-\frac{1}{2} \square p_{A B}$, so these equations do indeed hold.

Case $N \geq 3$. Now that the delicate balance of the case $N=2$ is upset, it is easy to see that, in particular, $J$ is forced to vanish. The more difficult thing to establish is that $J=0$ is sufficient to guarantee a solution of the equations as given in the theorem of $\S 5$. In the non-Abelian case this is a very tedious verification, but in the Abelian case there is a short cut. A Maxwell field may be split into self-dual and anti-self-dual parts given by potentials $\phi_{a}^{+}$and $\phi_{a}^{-}$satisfying $D_{(A}^{A^{\prime}} \phi_{B) A^{\prime}}^{+}=0$ and $D_{\left(A^{\prime}\right.}^{A} \phi_{\left.B^{\prime}\right) A}^{-}=0$. It is therefore clear that

$\Phi_{a}=\phi_{a}^{+}(x+\sigma)+\phi_{a}^{-}(x-\sigma), \quad \Psi_{A}^{j}=2 \tilde{\theta}^{A^{\prime} j} \phi_{A A^{\prime}}^{+}(x+\sigma), \quad \tilde{\Psi}_{A^{\prime} j}=2 \theta_{j}^{A} \phi_{A A^{\prime}}^{-}(x-\sigma)$

solve Witten's equations. By expanding these functions as power series in $\sigma$, it is elementary to change gauge so that $\theta_{j}^{A} \Psi_{A}^{j}+\tilde{\theta}^{A^{\prime} j} \tilde{\Psi}_{A^{\prime} j}=0$. This results in the formulae of $\S 5$.

\section{REFERENCES}

1. M. Batchelor, The structure of supermanifolds, Trans. Amer. Math. Soc. 253 (1979), 329388 .

2. N. P. Buchdahl, Analysis on analytic spaces and non-self-dual Yang-Mills fields, Trans. Amer. Math. Soc. 288 (1985), 431-469.

3. M. G. Eastwood, Ambitwistors, Twistor Newsletter 9 (1979), 55-58.

4. M. G. Eastwood, R. Penrose, and R. O. Wells, Jr., Cohomology and massless fields, Comm. Math. Phys. 78 (1981), 305-351.

5. M. G. Eastwood, The generalized Penrose-Ward transform, Math. Proc. Cambridge Philos. Soc. 97 (1985), 165-187.

6. M. G. Eastwood and C. R. LeBrun, Thickenings and supersymmetric extensions of complex manifolds, Amer. J. Math. 108 (1986), 1177-1192.

7. A. Ferber, Supertwistors and conformal supersymmetry, Nuclear Phys. B 132 (1978), 5564.

8. G. Fischer, Complex analytic geometry, Lecture Notes in Math., vol. 538, Springer, Berlin, 1976.

9. P. S. Green, J. Isenberg, and P. B. Yasskin, Non-self-dual gauge fields, Phys. Lett. B 78 (1978), 462-464.

10. P. S. Green, On holomorphic graded manifolds, Proc. Amer. Math. Soc. 85 (1982), 587-590.

11. J. Harnad, J. Hurtubise, M. Légaré, and S. Shnider, Constraint equations and field equations in supersymmetric $N=3$ Yang-Mills theory, Nuclear Phys. B 256 (1985), 609-620.

12. G. M. Henkin and Yu. I. Manin, Twistor description of classical Yang-Mills-Dirac fields, Phys. Lett. B 95 (1980), 405-408.

13. B. Kostant, Graded manifolds, graded Lie theory, and prequantization, Differential Geometric Methods in Mathematical Physics (Eds., K. Bleuler and A. Reetz), Lecture Notes in Math., vol. 570, Springer, Berlin, 1977, pp. 177-306.

14. Yu. I. Manin, Gauge fields and holomorphic geometry, J. Soviet Math. 21 (1983), 465-507.

15. R. Penrose, Applications of negative dimensional tensors, Combinatorial Mathematics and its Applications (Ed., D. J. A. Welsh), Academic Press, London and New York, 1971, pp. 221-244. 
16. _ Twistor theory, its aims and achievements, Quantum Gravity: An Oxford Symposium, Clarendon Press, Oxford, 1975, pp. 268-407.

17. R. Penrose and R. S. Ward, Twistors for flat and curved space-time, General Relativity and Gravitation (Ed., A. Held), Vol. II, Plenum Press, New York and London, 1980, pp. 283-328.

18. R. Penrose and W. Rindler, Spinors and spacetime, Cambridge Univ. Press, 1984.

19. R. Pool, Yang-Mills fields and extension theory, Mem. Amer. Math. Soc. (to appear).

20. M. Roček, An introduction to superspace and supergravity, Superspace and Supergravity (Eds., S. W. Hawking and M. Roček), Cambridge Univ. Press, 1981, pp. 71-131.

21. R. S. Ward, On self-dual gauge fields, Phys. Lett. A 61 (1977), 81-82.

22. R. O. Wells, Jr., Complex manifolds and mathematical physics, Bull. Amer. Math. Soc. (N.S.) 1 (1979), 296-336.

23. __ Complex geometry in mathematical physics, Les Presses de l'Université de Montréal, 1982.

24. E. Witten, An interpretation of classical Yang-Mills theory, Phys. Lett. B 77 (1978), 394-398.

25. B. G. Wybourne, Symmetry principles and atomic spectroscopy, Wiley-Interscience, New York, 1970.

Department of Pure Mathematics, University of Adelaide, GPo Box 498, Adelaide, S.A. 5001, Australia 